\title{
Handbook of
}

Porphyrin Science with Applications to Chemistry, Physics, Materials Science, Engineering, Biology and Medicine

-

Volume 29

Porphyrias and Sideroblastic Anemias

Edited by

Gloria C. Ferreira

Karl M. Kadish - Kevin M. Smith - Roger Guilard 


\section{Handbook of Porphyrin Science}

with Applications to Chemistry, Physics,

Materials Science, Engineering, Biology

and Medicine 


\title{
HANDBOOK OF PORPHYRIN SCIENCE with Applications to Chemistry, Physics, Materials Science, Engineering, Biology and Medicine
}

ISSN: 1793-9518

Editors: Karl M. Kadish (University of Houston, USA),

Kevin M. Smith (Louisiana State University, USA) and

Roger Guilard (Université de Bourgogne, France)

\author{
Vol. 1 Supramolecular Chemistry \\ Vol. 2 Synthesis and Coordination Chemistry \\ Vol. 3 Synthetic Methodology \\ Vol. 4 Phototherapy, Radioimmunotherapy and Imaging \\ Vol. 5 Heme Proteins
}

Vol. 6 NMR and EPR Techniques

Vol. 7 Physicochemical Characterization

Vol. 8 Open-Chain Oligopyrrole Systems

Vol. 9 Electronic Absorption Spectra - Phthalocyanines

Vol. 10 Catalysis and Bio-Inspired Systems — Part I

Vol. 11 Catalysis and Bio-Inspired Systems — Part II

Vol. 12 Applications

Vol. 13 Synthesis and Structural Studies

Vol. 14 Theoretical, Electron Transfer and Physical Studies

Vol. 15 Biochemistry of Tetrapyrroles

Vol. 16 Synthetic Developments - Part I

Vol. 17 Synthetic Developments - Part II

Vol. 18 Applications and Materials

Vol. 19 Biochemistry of Tetrapyrroles — Part II

Vol. 20 Chlorophylls and Related Systems

Vol. 21 Catalysis

Vol. 22 Biophysical and Physicochemical Studies of Tetrapyrroles

Vol. 23 Synthesis

Vol. 24 Coordination Chemistry and Materials

Vol. 25 Vitamin B12 and Related Systems 
Continued from previous page

Vol. 26 Heme Biochemistry

Vol. 27 Erythropoiesis, Heme and Applications to Biomedicine

Vol. 28 Chlorophyll, Photosynthesis and Bio-inspired Energy

Vol. 29 Porphyrias and Sideroblastic Anemias

Vol. 30 Heme Proteins - Part II 
This page intentionally left blank 


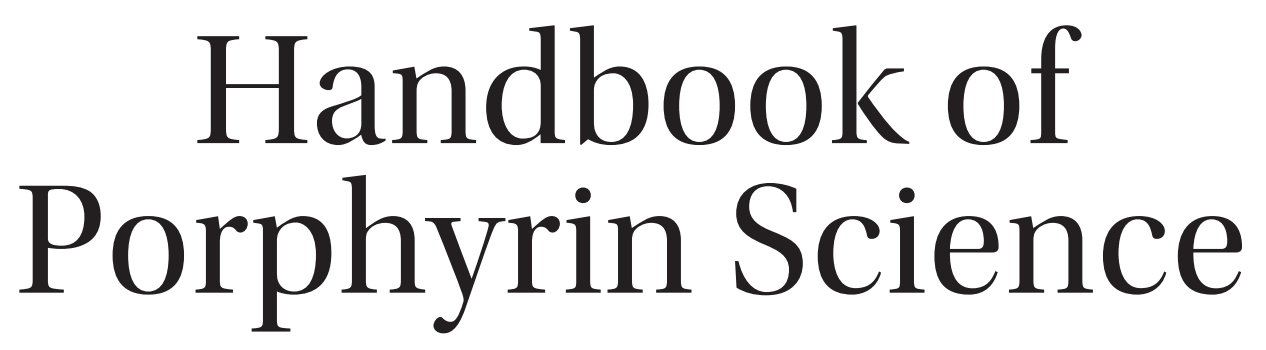

with Applications to Chemistry, Physics, Materials Science, Engineering, Biology and Medicine

\author{
-అయా \\ Volume 29 \\ Porphyrias and Sideroblastic Anemias
}

Editors

Gloria C. Ferreira

University of South Florida, USA

Karl M. Kadish

University of Houston, USA

Kevin M. Smith

Louisiana State University, USA

Roger Guilard

Université de Bourgogne, France 


\title{
Published by
}

World Scientific Publishing Co. Pte. Ltd.

5 Toh Tuck Link, Singapore 596224

USA office: 27 Warren Street, Suite 401-402, Hackensack, NJ 07601

UK office: 57 Shelton Street, Covent Garden, London WC2H 9HE

Library of Congress Control Number: 2011290361

\section{British Library Cataloguing-in-Publication Data}

A catalogue record for this book is available from the British Library.

\author{
HANDBOOK OF PORPHYRIN SCIENCE \\ with Applications to Chemistry, Physics, Materials Science, Engineering, Biology and Medicine \\ (Volumes 26-30) \\ Copyright @ 2014 by World Scientific Publishing Co. Pte. Ltd. \\ All rights reserved. This book, or parts thereof, may not be reproduced in any form or by any means, electronic \\ or mechanical, including photocopying, recording or any information storage and retrieval system now known \\ or to be invented, without written permission from the Publisher.
}

For photocopying of material in this volume, please pay a copying fee through the Copyright Clearance Center, Inc., 222 Rosewood Drive, Danvers, MA 01923, USA. In this case permission to photocopy is not required from the publisher.

ISBN 978-981-4407-74-8 (Set)

ISBN 978-981-4407-80-9 (Vol. 29)

Typeset by Stallion Press

Email: enquiries@stallionpress.com 


\section{Contents}

Preface for Volumes 26-30

xxi

Preface for Volumes 21-25

xxiii

Contributing Authors for Volumes 1-30

$\mathrm{xxV}$

Contents of Volumes $1-30$

liii

\section{3 / The Structural Basis of Porphyrias - Defects of Heme Biosynthetic Enzymes \\ Anna-Lena Kaufholz, Gunhild Layer, Dirk Heinz, Martina Jahn and Dieter Jahn}

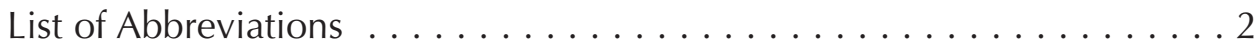

I. Introduction ......................... 3

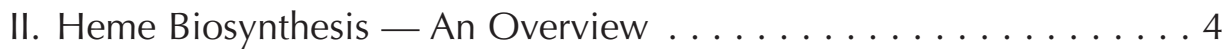

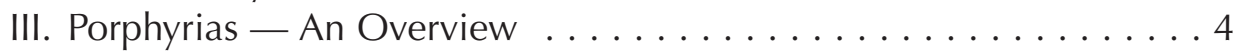

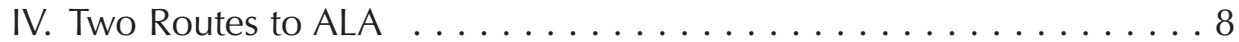

A. Shemin Pathway .................... 8

1. 5-Aminolevulinic Acid Synthase . . . . . . . . . . . 9

a. X-linked sideroblastic anemia . . . . . . . . . . . 10

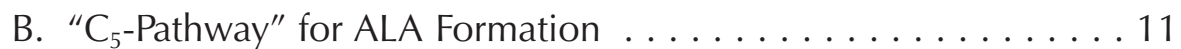

1. Glutamyl-tRNA Reductase . . . . . . . . . . . . . . 12

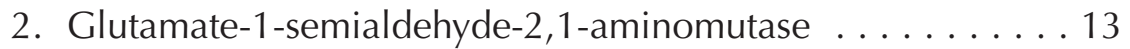

V. UROGEN Formation from ALA . . . . . . . . . . . . . . . 13

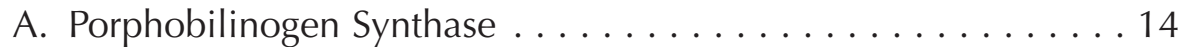

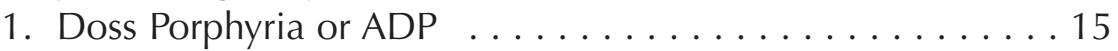

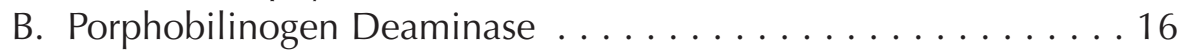

1. Acute Intermittent Porphyria . . . . . . . . . . . . 18

C. Uroporphyrinogen III Synthase $\ldots \ldots \ldots \ldots \ldots \ldots \ldots \ldots$

1. CEP or Gunther Disease . . . . . . . . . . . . . . . . . . 19

VI. Conversion of UROGEN into Heme . . . . . . . . . . . . 20

A. Uroporphyrinogen III Decarboxylase . . . . . . . . . . 20

1. PCT and Hepatoerythropoietic Porphyria ... . . . . . 23

B. Coproporphyrinogen III Oxidase .............. 23

1. HCP and Harderoporphyria ............... . 25 


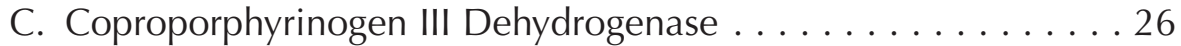

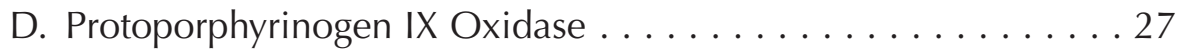

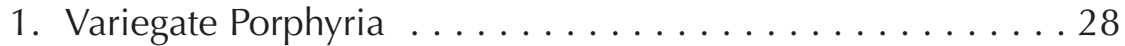

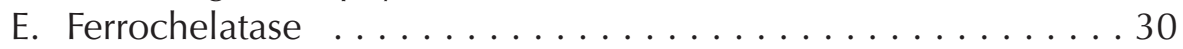

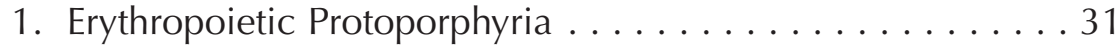

VII. Acknowledgments . . . . . . . . . . . . . . . . . . . 32

VIII. References ......................... 32

\section{4 / Sideroblastic Anemias: Molecular Basis, Pathophysiology, and Clinical Aspects}

\section{Sylvia S. Bottomley and Mark D. Fleming}

List of Abbreviations . . . . . . . . . . . . . . . . . . . . . . . . . . 44

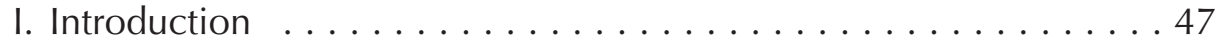

A. Historical Aspects . . . . . . . . . . . . . . . . 47

B. Definition of Sideroblastic Anemia . . . . . . . . . . . 48

C. Classification of Sideroblastic Anemias . . . . . . . . . . . . 49

II. Central Roles of the Mitochondrion in the Pathogenesis of Sideroblastic Anemias . . . . . . . . . . . . . . . . . 50

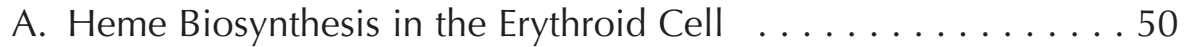

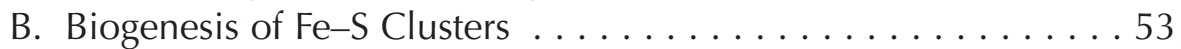

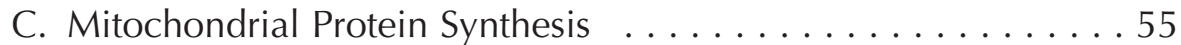

D. Iron Homeostasis in the Erythroid Cell . . . . . . . . . . . . 56

III. Congenital Sideroblastic Anemias . . . . . . . . . . . . . 57

A. X-Linked Sideroblastic Anemia . . . . . . . . . . . . . . 57

1. Molecular Basis and Pathogenesis . . . . . . . . . . 57

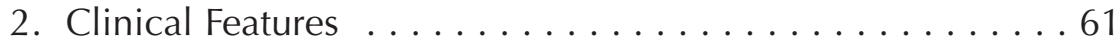

3. Treatment and Prognosis . . . . . . . . . . . 62

B. Autosomal Recessive Sideroblastic Anemias . . . . . . . . . . 63

1. Molecular Basis and Pathogenesis . . . . . . . . . . 63

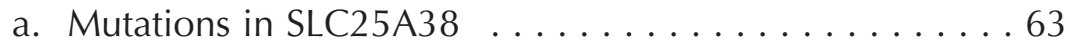

b. GLRX5 Deficiency ................... 64

c. Erythropoietic Protoporphyria . . . . . . . . . . 65

2. Clinical Features ...................665

3. Treatment and Prognosis . . . . . . . . . . . . 65

C. Undefined Category of Congenital Sideroblastic

Anemia(s) . . . . . . . . . . . . . . . . . .666

IV. Syndromic Forms of Congenital Sideroblastic Anemia . . . . . . 66

A. X-Linked Sideroblastic Anemia with Ataxia . . . . . . . . . . 66

B. Myopathy, Lactic Acidosis, and Sideroblastic Anemia . . . . . . 67 
C. Congenital Sideroblastic Anemia, B Cell Immunodeficiency, Periodic Fevers, and Developmental Delay . . . . . . . . 68

D. Pearson Marrow-pancreas Syndrome . . . . . . . . . . . . . 68

E. Thiamine Responsive Megaloblastic Anemia . . . . . . . . . . 69

V. Acquired Clonal Sideroblastic Anemia . . . . . . . . . . . . 70

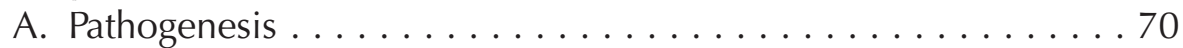

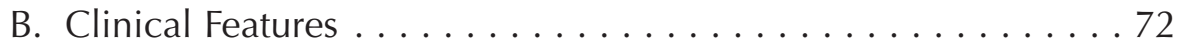

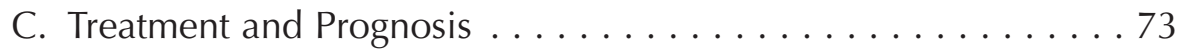

VI. Acquired Reversible Sideroblastic Anemias . . . . . . . . . . . . . 73

A. Alcoholism . . . . . . . . . . . . . . . . . . . . . . 73

B. Drug-induced Sideroblastic Anemia . . . . . . . . . . . . . 74

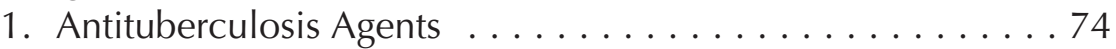

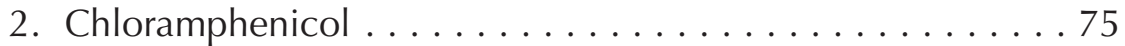

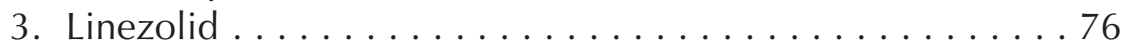

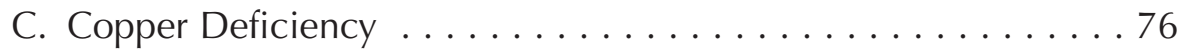

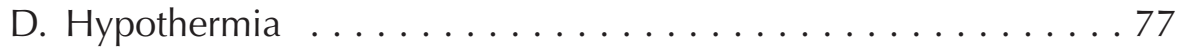

VII. Summary and Future Prospects . . . . . . . . . . . . . 77

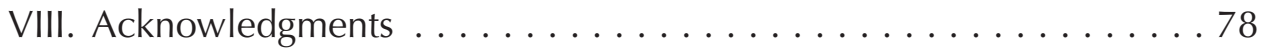

IX. References .......................78

\section{5 / Heme Biosynthesis and Pathophysiology of Porphyrias} Zoubida Karim, Laurent Gouya, Jean-Charles Deybach and Hervé Puy

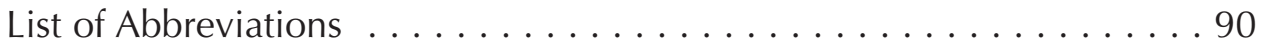

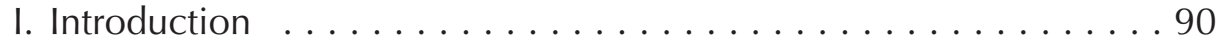

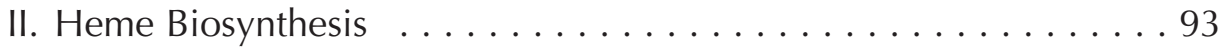

A. Structure of Porphyrins $\ldots \ldots \ldots \ldots \ldots \ldots \ldots \ldots \ldots \ldots$

B. Synthesis of Porphyrin Precursors: $\delta$-ALA and PBG . . . . . 94

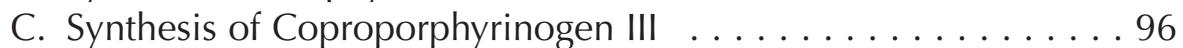

D. Synthesis of Heme . . . . . . . . . . . . . . . 96

III. Excretion of Porphyrin Precursors and Porphyrins . . . . . . . 99

A. Porphyrin Precursors and Porphyrins in Urine . . . . . . . 99

1. Porphyrin Precursors . . . . . . . . . . . . . 99

2. Porphyrins ...................... 99

B. Porphyrin Precursors and Porphyrins in Feces . . . . . . . . 101

IV. Heme Catabolism . . . . . . . . . . . . . . . . . . . . . . 103

V. Regulation of Heme Synthesis . . . . . . . . . . . . . . 104

A. In the Liver . . . . . . . . . . . . . . . . . . . . . . . 105

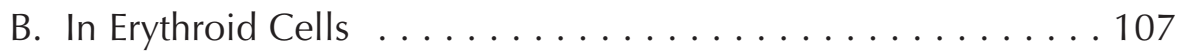


VI. Pathogenesis of an Acute Attack . . . . . . . . . . . . . . 112

VII. Pathogenesis of Skin Injury . . . . . . . . . . . . . . . 113

VIII. Conclusions and Future Directions . . . . . . . . . . . . . 114

IX. References ........................... 115

\section{6 / Clinical and Molecular Epidemiology of the Porphyrias} Michael N. Badminton, George H. Elder and Sharon D. Whatley

List of Abbreviations . . . . . . . . . . . . . . . . . . . 120

I. Introduction ........................ 121

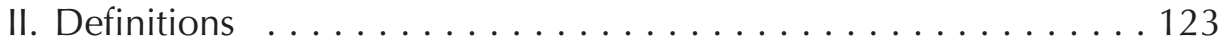

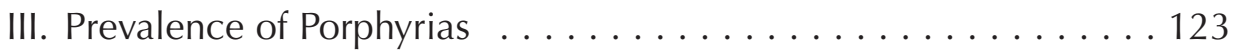

IV. Autosomal Dominant Acute Porphyrias . . . . . . . . . . . 126

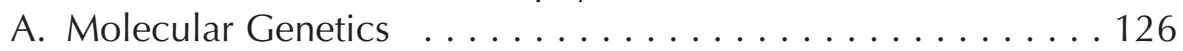

1. HMBS, CPOX, or PPOX Mutations on One Allele . . . . 126

2. Allelic Heterogeneity . . . . . . . . . . . . . 128

3. HMBS, CPOX, or PPOX Mutations on Both Alleles ..... 128

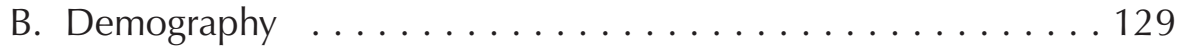

C. Prevalence and Incidence . . . . . . . . . . . . . . . . 130

1. Acute Intermittent Porphyria . . . . . . . . . . . . 130

2. VP and HCP ... . . . . . . . . . . . . . . . . . . 131

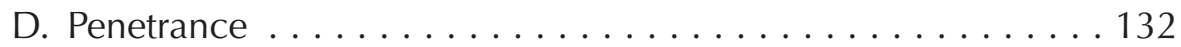

V. Porphyria Cutanea Tarda . . . . . . . . . . . . . . . . . 134

A. Molecular Genetics of PCTF . . . . . . . . . . . . . 134

1. UROD Mutations on One Allele . . . . . . . . . . . . 134

2. UROD Mutations on Both Alleles . . . . . . . . . . 135

B. Demography . . . . . . . . . . . . . . . . . . . 135

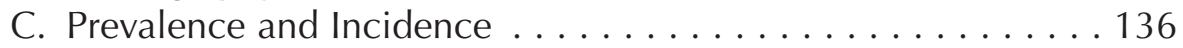

D. Susceptibility Factors . . . . . . . . . . . . . . . . 136

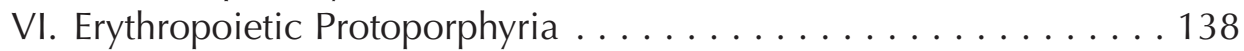

A. Molecular Genetics . . . . . . . . . . . . . . . . . . 138

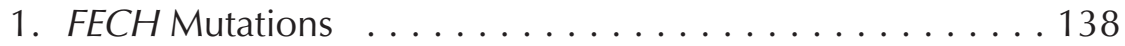

2. FECH IVS3-48C Allele . . . . . . . . . . . . . . . 139

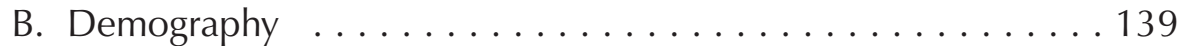

C. Prevalence and Incidence . . . . . . . . . . . . . . 139

VII. Conclusion . . . . . . . . . . . . . . . . . . . . . . . . . . . . . . . . . 142

VIII. References ... . . . . . . . . . . . . . . . . . . . . . . 142 


\section{7 / Congenital Erythropoietic Porphyria Jordi To-Figueras, Oscar Millet and Carmen Herrero}

List of Abbreviations . . . . . . . . . . . . . . . . . . . . . . . . . . . 152

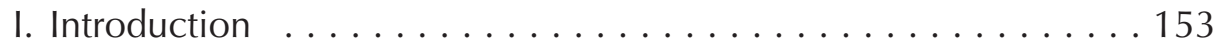

II. History . . . . . . . . . . . . . . . . . . . . . . 153

III. Core Defect in CEP . . . . . . . . . . . . . . . . 156

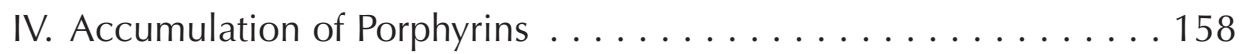

V. Clinical Features of CEP . . . . . . . . . . . . . . . . . . 161

A. Hematological Features . . . . . . . . . . . . . . . . 161

B. Cutaneous Manifestations . . . . . . . . . . . . . . . 163

C. Pathogenesis of Skin Lesions . . . . . . . . . . . . . 167

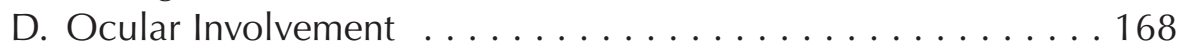

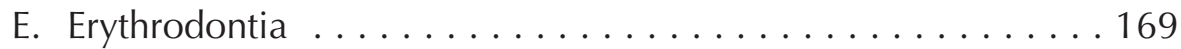

F. Skeletal Abnormalities . . . . . . . . . . . . . . . . . . 170

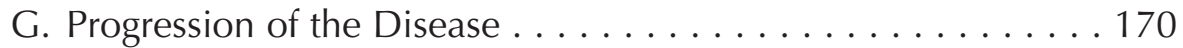

H. Association with Other Diseases . . . . . . . . . . . . . . 171

I. Autopsy Reports . . . . . . . . . . . . . . . . . . . . 172

J. Mild Cases . . . . . . . . . . . . . . . . . . . . . . . 172

K. Late-onset Cases . . . . . . . . . . . . . . . . . . . . . 173

L. Prenatal Manifestations of Severe Cases . . . . . . . . . . . 173

M. Neonatal Period . . . . . . . . . . . . . . . . . . . . 174

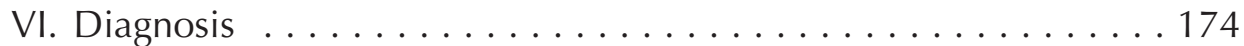

VII. CEP in Animals . . . . . . . . . . . . . . . . . . . . . . 180

VIII. URO Synthase Gene . . . . . . . . . . . . . . . . . . . 181

IX. Mutations Causing CEP . . . . . . . . . . . . . . . . . 183

A. Mutations in the URO Synthase Gene . . . . . . . . . 183

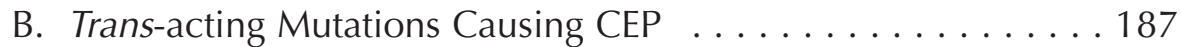

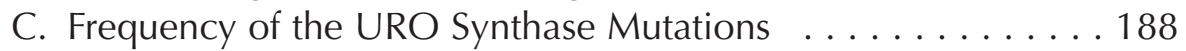

X. Relationship between the Genotype and the Clinical Phenotype. . 188

A. Homozygous Patients . . . . . . . . . . . . . . . . . 189

B. Compound Heterozygous Patients . . . . . . . . . . . . 191

C. Modifier Genes in CEP . . . . . . . . . . . . . . . . . . . . 193

D. Mixed Phenotypes . . . . . . . . . . . . . . . . . . . 193

XI. Murine Models of CEP . . . . . . . . . . . . . . . . . . . . . . . 194

XII. URO Synthase Structure and Function . . . . . . . . . . . . . . . 195

A. URO Synthase High-Resolution Structures . . . . . . . . . . 195

B. Substrate-binding Site and URO Synthase Catalytic Mechanism 
C. Mapping of Missense Mutations in URO Synthase . . . . . 198

D. Stability and Catalytic Activity of CEP-associated URO Synthase Variants . . . . . . . . . . . . . . . . . . . . . 199

E. Structural Rescue of Deficient URO Synthase Activity . . . . . . 202 XIII. Treatment of CEP . . . . . . . . . . . . . . . . . . . . . . . 203

A. Miscellaneous . . . . . . . . . . . . . . . . . . 203

B. Hematopoietic Stem Cell Transplantation . . . . . . . . . . 205

C. Experimental Gene Therapy . . . . . . . . . . . . . . . . 207

XIV. Conclusions and Future Directions . . . . . . . . . . . . . . . . . 207

XV. Acknowledgments . . . . . . . . . . . . . . . . . . . . 208

XVI. References . . . . . . . . . . . . . . . . . . . . . . . . . . 208

\section{8 / Porphyria Cutanea Tarda Ashwani K. Singal and John D. Phillips}

List of Abbreviations . . . . . . . . . . . . . . . . . . . . . 220

I. Introduction ........................ 221

II. History and Nomenclature . . . . . . . . . . . . . . . . . . . . 221

III. Human Uroporphyrinogen Decarboxylase . . . . . . . . . 222

A. UROD Locus . . . . . . . . . . . . . . . . . 222

B. UROD Activity . . . . . . . . . . . . . . . . . . 223

C. Molecular Pathology and UROD Deficiency Classification . . . 224

IV. Pathogenesis . . . . . . . . . . . . . . . . . . . . 225

A. Experimental Uroporphyria . . . . . . . . . . . . . 225

B. Iron Overload . . . . . . . . . . . . . . . . . . . 226

C. Cytochrome P4501A2 Induction and Polymorphisms . . . . 227

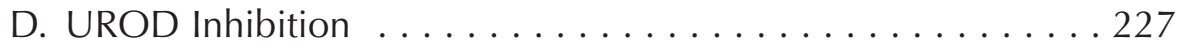

E. Susceptibility Factors . . . . . . . . . . . . . . . . . 228

1. Alcohol . . . . . . . . . . . . . . . . . . . . 228

2. Smoking ......................... . 229

3. Hepatitis C ........................ . . 229

4. HFE Mutations ... . . . . . . . . . . . . . . . . . 229

5. Human Immunodeficiency Virus (HIV) Infection . . . . . 231

6. Estrogens ....................... 231

7. Other Conditions . . . . . . . . . . . . . . . . . 231

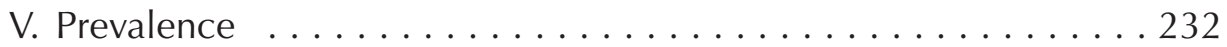

VI. Clinical Features . . . . . . . . . . . . . . . . . . 232

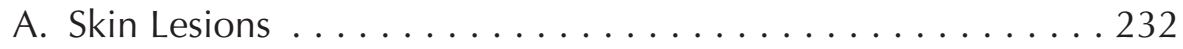

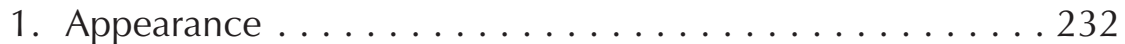

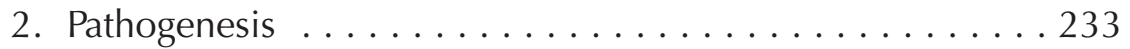


3. Histology . . . . . . . . . . . . . . . . . . 233

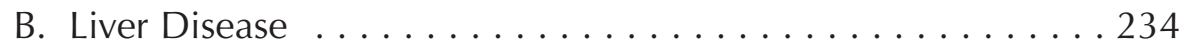

VII. Diagnosis . . . . . . . . . . . . . . . . . . . . . 235

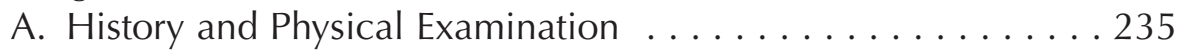

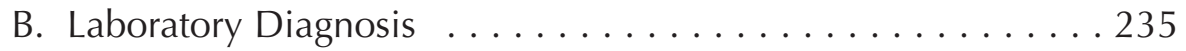

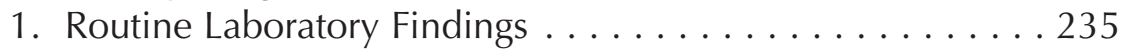

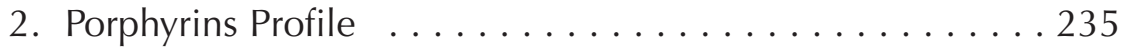

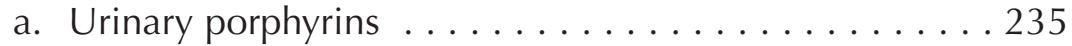

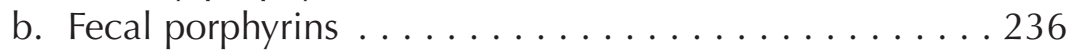

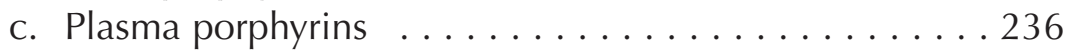

d. Hepatic porphyrins . . . . . . . . . . . . . . . . 237

3. Evaluation for Susceptibility Factors . . . . . . . . 237

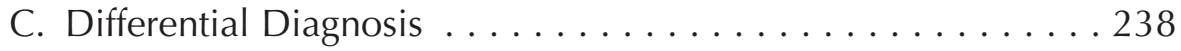

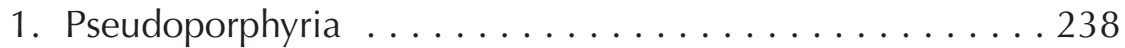

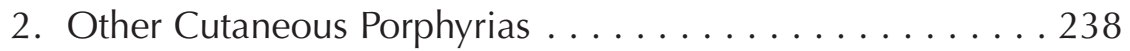

VIII. Treatment . . . . . . . . . . . . . . . . . . . . . . 240

A. General Measures . . . . . . . . . . . . . . . . . . . 240

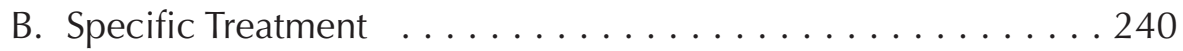

1. Repeated Phlebotomy . . . . . . . . . . . . . . . . . 240

2. 4-Aminoquinolines (Chloroquine and Hydroxychloroquine). . 243

3. Comparison of Phlebotomy and 4-Aminoquinolines . . . . 247

4. Other Treatment Options . . . . . . . . . . . . . . . 248

5. Treatment of PCT with Concomitant HCV Infection . . . . . 248

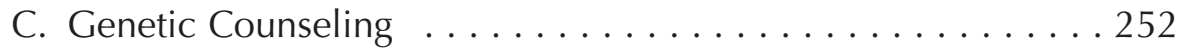

IX. Natural History and Prognosis . . . . . . . . . . . . . 252

X. Summary and Conclusions . . . . . . . . . . . . . . 253

XI. Acknowledgments . . . . . . . . . . . . . . . . . . 253

XII. References ............................ 253

\section{9 / Variegate Porphyria}

Peter Meissner, Anne Corrigall and Richard Hift

List of Abbreviations . . . . . . . . . . . . . . . . . . . . . . . . . . . . . 264

I. Introduction ........................ 264

II. History and Prevalence . . . . . . . . . . . . . . . 265

A. History . . . . . . . . . . . . . . . . . . . . . . 265

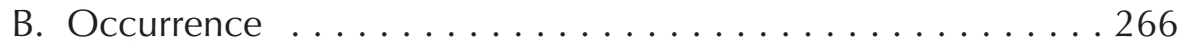

III. Heme Biosynthetic Enzymes in VP . . . . . . . . . . 266

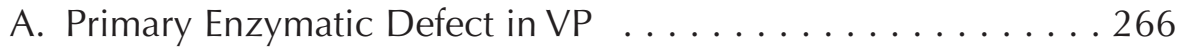

B. ALA Synthase and PBG Deaminase in VP . . . . . . . 267 


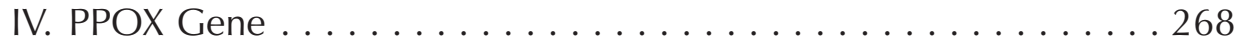

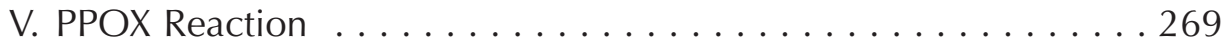

VI. PPOX Gene Product . . . . . . . . . . . . . . . . . . 270

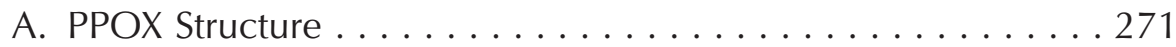

VII. PPOX Mutations . . . . . . . . . . . . . . . . . . . . . . . 272

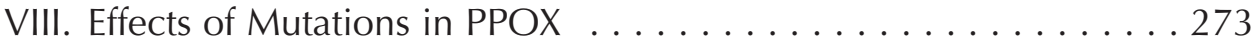

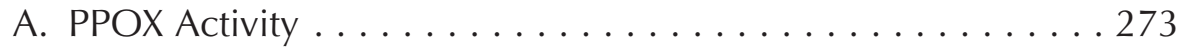

B. PPOX Structure ....................... . . 273

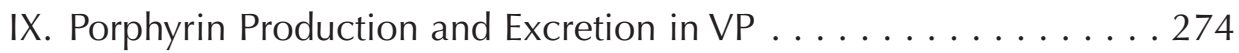

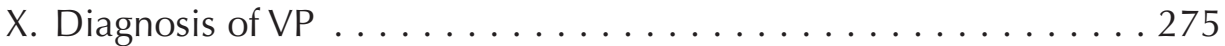

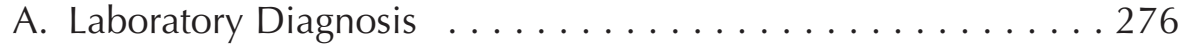

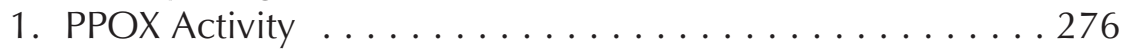

2. Porphyrin Analysis . . . . . . . . . . . . . . . . 276

3. Plasma Fluorescence Scanning . . . . . . . . . . . . . 278

4. Urinary Porphyrin Precursor Measurement . . . . . . . . 279

5. DNA Mutational Analysis . . . . . . . . . . . . . . . . 279

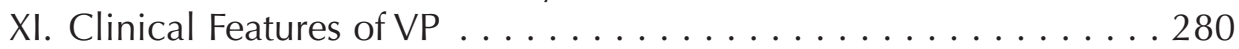

A. Skin Disease . . . . . . . . . . . . . . . . . . . . . 281

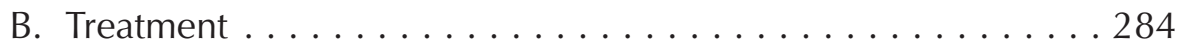

C. Acute Attack . . . . . . . . . . . . . . . . . . . . . . 284

D. Precipitants of the Acute Attack . . . . . . . . . . . . . 285

E. Treatment of the Acute Attack . . . . . . . . . . . . . . . 287

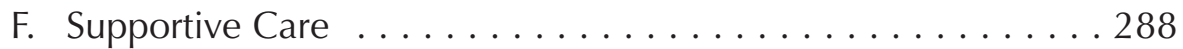

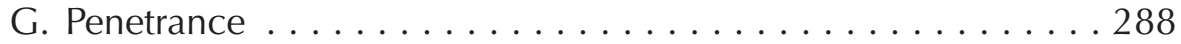

H. Homozygous VP . . . . . . . . . . . . . . . . . 289

XII. Final Comment and Future Directions . . . . . . . . . . . . . . . . 290

XIII. Acknowledgments . . . . . . . . . . . . . . . . . . . . . . . . 290

XIV. References . . . . . . . . . . . . . . . . . . . . . . . . . . . . . . 291

\section{0 / Erythropoietic Protoporphyria and X-Linked Dominant} Protoporphyria Xiaoye Schneider-Yin and Elisabeth I. Minder

List of Abbreviations . . . . . . . . . . . . . . . . . . . . . . . 300

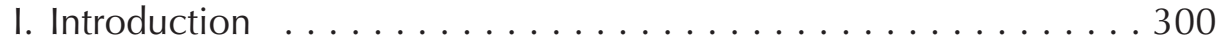

II. Prevalence and Epidemiology . . . . . . . . . . . . . . . . 301

III. Clinical Features . . . . . . . . . . . . . . . . . . . . . . . . 301

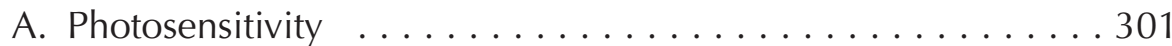

B. Hematologic Abnormalities . . . . . . . . . . . . . . . 304

C. Iron Metabolism in EPP . . . . . . . . . . . . . . . . 305 
D. Liver Involvement . . . . . . . . . . . . . . . . . . . . . . 306

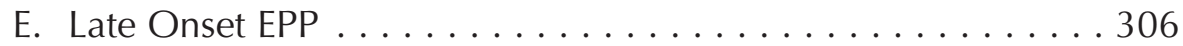

IV. Laboratory Findings . . . . . . . . . . . . . . . . 306

A. Plasma Fluorescence Scanning . . . . . . . . . . . . . . 307

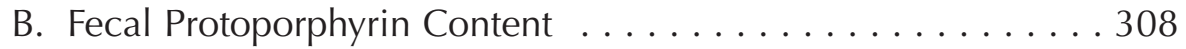

C. Zinc and Metal-free Protoporphyrin Concentrations in Erythrocytes . . . . . . . . . . . . . . . . . . 308

D. Ferrochelatase Activity Measurement and FECH

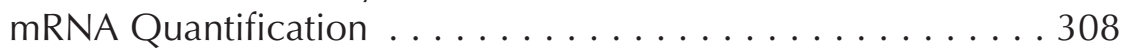

V. Inheritance and Genetic Defects . . . . . . . . . . . . . . 310

A. Erythropoietic Protoporphyria . . . . . . . . . . . . . 310

1. Autosomal Dominant Trait . . . . . . . . . . . . 311

2. Autosomal Recessive Trait . . . . . . . . . . . . 316

3. Genotype-Phenotype Correlation in EPP . . . . . . . . 317

B. X-Linked Dominant Protoporphyria . . . . . . . . . . . . 319

VI. Treatment Options . . . . . . . . . . . . . . . . . . . . . . 319

A. Conditioned Behavior and Efficacy Assessment . . . . . . 320

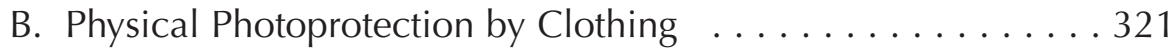

C. Medical Interventions to Prevent Phototoxicity . . . . . . . . 322

D. Protoporphyrin-Related Liver Disease . . . . . . . . . . 323

VII. Summary . . . . . . . . . . . . . . . . . . . . . . . . . . . . . 323

VIII. References . . . . . . . . . . . . . . . . . . . . . . . . . 324

\section{1 / Liver Transplantation in Acute Hepatic Porphyria and Erythropoietic Protoporphyria}

\section{Staffan Wahlin and Pauline Harper}

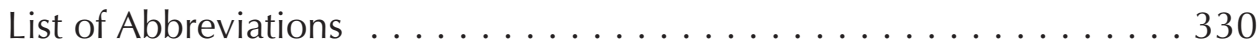

I. Introduction ....................... 331

II. Acute Hepatic Porphyria . . . . . . . . . . . . . . . . . . . 332

A. History of Liver Transplantation in Acute Hepatic Porphyria . . 334

B. Indications for Liver Transplantation . . . . . . . . . . . . 335

C. Conservative Treatment of Severe AIP . . . . . . . . . . . 336

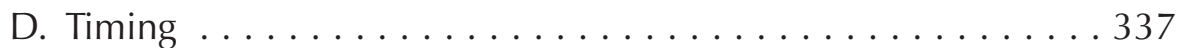

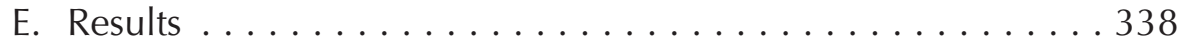

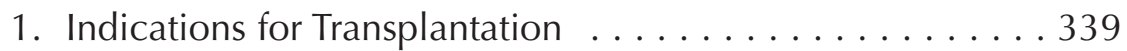

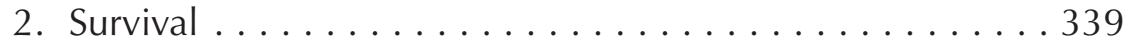

3. Clinical and Biochemical Results . . . . . . . . . . 339

4. Complications . . . . . . . . . . . . . . . . . 340

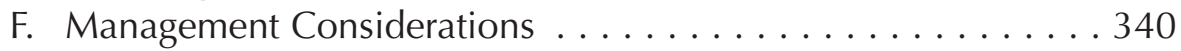


G. Combined Liver and Kidney Transplantation . . . . . . . . . . . . 341

H. Domino Transplantation . . . . . . . . . . . . . . . 341

I. Prelude to Liver Transplantation . . . . . . . . . . . . . . . 342

J. Perspectives for the Future . . . . . . . . . . . . . . . 345

III. Erythropoietic Protoporphyria . . . . . . . . . . . . . . . . 347

A. History of Liver Transplantation . . . . . . . . . . . . 348

B. Indications for Liver Transplantation . . . . . . . . . . . . 348

C. Results . . . . . . . . . . . . . . . . . . . . . . . 349

D. Complications . . . . . . . . . . . . . . . . . . 349

1. Recurrent Liver Disease . . . . . . . . . . . . . . . 350

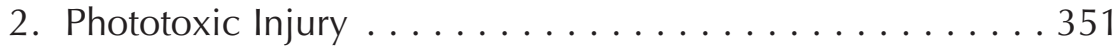

3. Neuropathy . . . . . . . . . . . . . . . . . . . 351

4. Biliary Tract Complications . . . . . . . . . . . . . . . . 352

E. Management Considerations . . . . . . . . . . . . . . 352

1. Preoperative Treatment . . . . . . . . . . . . . . . . 352

2. Perioperative Management . . . . . . . . . . . . . 354

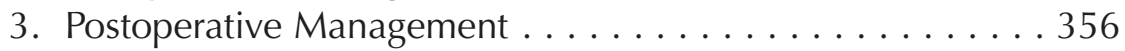

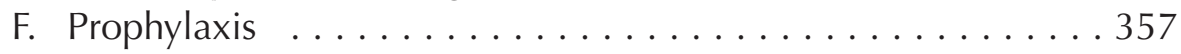

G. Follow-up . . . . . . . . . . . . . . . . . . . . . . . . 357

H. Hematopoietic Stem Cell Transplantation . . . . . . . . . . . 357

I. Perspectives for the Future . . . . . . . . . . . . . . 359

IV. Summary . . . . . . . . . . . . . . . . . . . . . 360

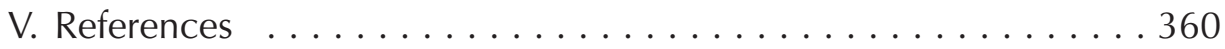

\section{2 / Clinical and Laboratory Diagnosis of the Porphyrias Karl E. Anderson}

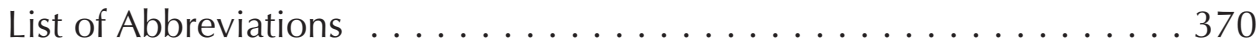

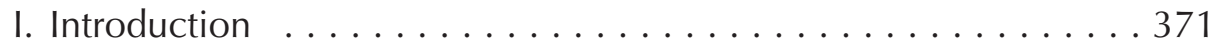

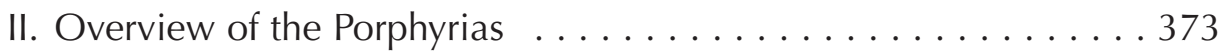

A. Heme Biosynthesis in the Porphyrias . . . . . . . . . . 373

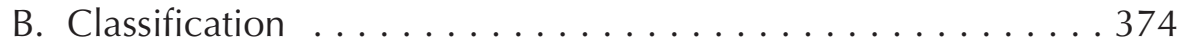

C. Enzyme Alterations and Inheritance . . . . . . . . . . 375

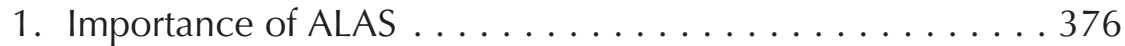

D. Genotype-Phenotype Correlations and Factors Modifying Disease Severity . . . . . . . . . . . . . . . . . . 377

E. Deficiencies of Heme Pathway Enzymes in Other Conditions . 378

III. Diagnostic Considerations in the Porphyrias . . . . . . . . . 379

A. Porphyrin Precursors and Porphyrins - Properties and Routes of Excretion . . . . . . . . . . . . . . . . . . 379 
B. Three Most Common Porphyrias . . . . . . . . . . . 380

C. A Staged Approach to Laboratory Diagnosis of Porphyrias . . . 381

IV. Acute Porphyrias . . . . . . . . . . . . . . . . . . 381

A. Clinical Features and Indications for Testing . . . . . . . 382

B. First-line Testing for Acute Porphyrias . . . . . . . . . . 384

C. Second-line Testing for Acute Porphyrias . . . . . . . . . . 387

D. Additional Testing When Urine PBG is Normal . . . . . . . . 389

E. Testing for ADP and Other Causes of ALAD Deficiency . . . . 389

F. Other Laboratory Findings in Acute Porphyrias . . . . . . . . 390

V. Blistering Cutaneous Porphyrias . . . . . . . . . . . . 391

A. Clinical Features and Indications for Testing . . . . . . . . . 391

1. Porphyria Cutanea Tarda . . . . . . . . . . . . . . . . . . 391

2. Hepatoerythropoietic Porphyria . . . . . . . . . . . . 393

3. Congenital Erythropoietic Porphyria . . . . . . . . . . 393

4. Hereditary Coproporphyria . . . . . . . . . . . . . . . . 395

5. Variegate Porphyria . . . . . . . . . . . . . . . 395

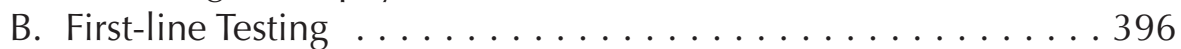

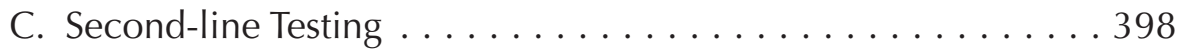

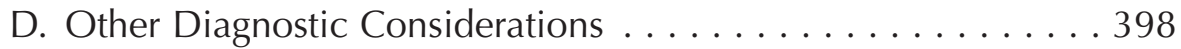

1. Dermatopathology ... . . . . . . . . . . . . . . . . 399

2. Liver Function and Histopathology . . . . . . . . . . . . . 399

3. Other Laboratory Abnormalities and Disease Associations . . 399

VI. Non-Blistering Photosensitivity — EPP . . . . . . . . . . . . 399

A. Clinical Features and Indications for Testing . . . . . . . . 400

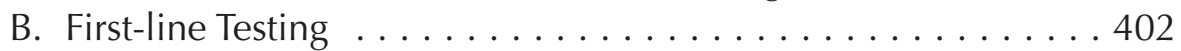

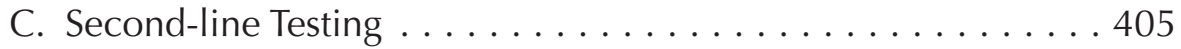

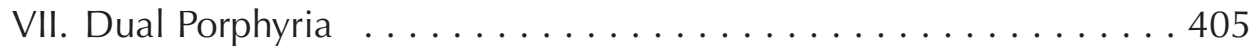

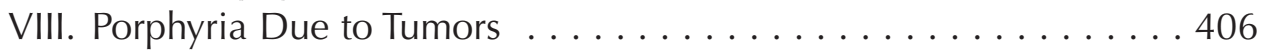

IX. Acknowledgments .....................4406

X. References ........................ 406

\section{3 / Liver Gene Therapy Approaches for Acute Intermittent} Porphyria: Metabolic Correction and Immunological Hurdles Antonio Fontanellas and Ignacio Melero

List of Abbreviations . . . . . . . . . . . . . . . . . . . . 416

I. Introduction and Current Therapies . . . . . . . . . . . . 417

II. Animal Models . . . . . . . . . . . . . . . . . . . . . . . 419

III. Enzyme Replacement Therapy . . . . . . . . . . . . 421

IV. Liver Gene Therapy . . . . . . . . . . . . . . . . . . 421 
A. Non-viral Delivery Systems . . . . . . . . . . . . . . . . . . 423

B. Viral Vectors . . . . . . . . . . . . . . . . . . . . . . . . . . . 425

1. Recombinant Adenoviral Vectors . . . . . . . . . . . . . 425

2. Recombinant Adeno-Associated Viral Vectors . . . . . . . 427

V. Preclinical Studies with rAAV Vectors . . . . . . . . . . . . 430

A. Development of a Safe and Manufacturing Process of rAAV Vectors for Clinical Studies . . . . . . . . . . . . . . 432

B. Preclinical Toxicology Studies . . . . . . . . . . . . . . 434

C. Potential Risk Concerns . . . . . . . . . . . . . . . . . . . 434

1. Recombination with a Wild-type Virus . . . . . . . 434

2. Insertional Mutagenesis . . . . . . . . . . . . . . 435

3. Germline Transmission . . . . . . . . . . . . . 435

4. Immunogenicity of the Vector . . . . . . . . . . . . 436

a. Pre-existing immunity to the wild-type AAVs . . . . 446 436

b. Adaptive immune response to rAAV vectors . . . . . . . 437

c. Readministration with the same rAAV vector . . . . . . 441

d. Immunosuppresion protocol in acute porphyrias . . . . 442

5. Immune Response to the Transgene Protein . . . . . . . . . 443

VI. Future Directions and Concluding Remarks . . . . . . . . . . . 444

VII. Acknowledgments . . . . . . . . . . . . . . . . . . . . . 445

VIII. References ... . . . . . . . . . . . . . . . . . . . . . . . 445

\section{4 / Cellular and Gene Therapy for Erythropoietic Porphyrias} Emmanuel Richard, Cécile Ged, Aurélie Bedel, François Moreau-Gaudry and Hubert de Verneuil

List of Abbreviations . . . . . . . . . . . . . . . . . . . . . . 452

I. Introduction: Molecular Basis for Erythropoietic Porphyrias . . . . 453

II. Animal Models of Erythropoietic Porphyrias . . . . . . . . . 456

A. Animal Models of Erythropoietic Protoporphyria . . . . . . 456

B. Animal Models of Congenital Erythropoietic Porphyria . . . . . 457

C. Animal Models of Porphyria Cutanea Tarda and

Hepatoerythropoietic Porphyria . . . . . . . . . . . . . . 460

III. Allogeneic Bone Marrow Transplantation for Erythropoietic Porphyrias . . . . . . . . . . . . . . . 461

A. Allogeneic Bone Marrow Transplantation for Congenital Erythropoietic Porphyria . . . . . . . . . . . . . . . . . 461

B. Allogeneic Bone Marrow Transplantation for Erythropoietic Protoporphyria. . . . . . . . . . . . . . . . . . . . . . . . 465

IV. Ex Vivo Hematopoietic Stem Cell Gene Therapy . . . . . . . . . . 469 
A. Considerations on Cells and Vectors for Hematopoietic Stem Cell Gene Therapy of Erythropoietic Porphyrias . . . . . . 469

1. Hematopoietic Stem Cell Origin and Ex Vivo Culture ... 469

2. Therapeutic Vector Design. . . . . . . . . . . . . . . . . . . . 469

B. Gene Therapy for Hepatoerythropoietic Porphyria . . . . . . . 472

C. Gene Therapy for Congenital Erythropoietic Porphyria . . . . 472

D. Gene Therapy for Erythropoietic Protoporphyria . . . . . . . . 474

V. Perspectives in Regenerative Medicine for Erythropoietic

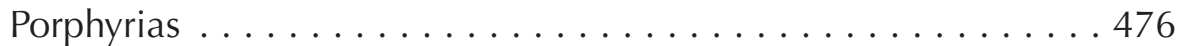

A. Progress in Stem Cell Source: Induced Pluripotent Stem Cells . . . . . . . . . . . . . . . . . . . . . . 476

B. Progress in Gene Therapy: Targeted Gene Correction . . . . . . . 478 VI. Conclusions . . . . . . . . . . . . . . . . . . . . . . . 479

VII. References ............................ . 479

Index to Volume $29 \ldots \ldots$. . . . . . . . . . . . . . . . . . . . . 485 
This page intentionally left blank 


\section{Preface for Volumes 26-30}

It was with a certain trepidation that I embarked on this project of organizing and editing a five-volume set on Porphyrin Biochemistry with an emphasis on Applications to Biomedicine and Bio-inspired Energy. While the accelerated research developments and expanded applied interest in the biochemistry of porphyrins and natural porphyrinoid pigments during this past decade made it a particularly opportune time to assess these developments, three issues concerned me: 1) Would there be a readership?, 2) What would be the best forum to implement such a project?, and 3) Would leading investigators contribute with comprehensive chapters? After pondering these questions, I realized that despite the explosion of printed journals and electronic media and platforms as vehicles for scientific information, the pursuit of science will always depend on serious review of present and past literature. Based on this premise, I assured myself that there should be an eager "Porphyrin Science" readership, who not only have the same passion for porphyrins, but will also propagate interest in the mysteries and intricacies of the "porphyrin world" to younger and newer investigators. Probably now more than ever, scientists need help in foraging through the mass of literature in order to read broadly yet be selective and discern among the publishable material, which, unfortunately, does not always meet scientific rigor. Thus, my second concern was quickly dispelled when I found that my 'project' could be incorporated into a fivevolume set (Volumes 26-30) in the "Handbook of Porphryrin Science", which consistently has been the premier forum in the dissemination of the porphyrin and tetrapyrrole research and applications for the past two decades.

With the constant pressure to secure funding in this present era of tight budgets, scientists find themselves 'time-limited' and, understandably, reluctant to take on additional activities, such as developing and writing a comprehensive and upto-date scientific review. And so my third concern lingered until I received the replies to my invitations. To my delight, the vast majority of the invited scientists accepted the invitation, or more bluntly, the challenge.

I am indebted to the authors of the chapters in this five-volume set for their generosity with time, their dedication and commitment to this project, the breadth of their reviews and lastly, but so importantly, adherence to deadlines. Clearly, it was their effort that has converted the idea of this project into reality. I hope that 
the reader will enjoy the chapters as much as I did and will participate in spreading the knowledge of porphyrin science to the broader scientific community.

Finally, I would like to gratefully acknowledge Professors Karl Kadish, Kevin Smith and Roger Guilard for allowing me to organize and edit Volumes 26-30 of the "Handbook of Porphryrin Science", and I especially thank Professors Smith and Kadish for their encouragement, help, advice and prompt replies to my numerous emails. A big thank you goes to the copy editors, production team and World Scientific Publishing Company for their tireless work and expertise that brought this project to fruition.

Gloria C. Ferreira (Tampa, Florida, USA) July 11,2013 


\section{Preface for Volumes 21-25}

As both researchers and editors in the field of porphyrin and phthalocyanine science, we are delighted to see that new investigators in the field are establishing themselves, and acquiring well-deserved leadership credentials. When we started this project, we suspected, as editors, that a 20 volume series of the Handbook would "do the job", at least for the near and middle term. However, this was not the case, since many of the new investigators who have entered this research area were not content to simply extend the field of tetrapyrrole research and applications, but rather have chosen to develop new areas upon which they are now stamping their imprint. So here we are again, with another five (and eventually ten) new volumes of the Handbook of Porphyrin Science. The field continues to expand, especially with regard to porphyrin and phthalocyanine applications; at the same time the population of research-active "porphyrinologists" has been increasing at a very gratifying rate, particularly in Asia. In the $21^{\text {st }}$ Century, porphyrin and tetrapyrrole science is alive, well, and expanding, and this is abundantly obvious in the new Volumes 21-25 of the Handbook.

As always we are very grateful to our authors - both new and established investigators - for providing excellent chapters on all that is new with tetrapyrroles. Though we as editors are now adept at gathering promised contributions, it still takes diligent and talented authors to produce them. We thank them all.

We would also like to thank our production team at World Scientific Publishing Company (WSPC) for all their fine work. They care about the final product just as much as we do. And finally, we have to voice our appreciation of the senior management at WSPC for continuing to believe, along with us, in the importance of porphyrin science.

Karl M. Kadish (Houston)

Kevin M. Smith (Baton Rouge)

Roger Guilard (Dijon)

April 1, 2012 
This page intentionally left blank 


\section{Contributing Authors for Volumes 1-30*}

\section{Roger Alberto}

University of Zürich

CH-8057 Zürich

Switzerland

Chapter 116

\section{Hasrat Ali}

Université de Sherbrooke

Sherbrooke, Québec, Canada

Chapter 16

\section{Salam Al-Karadaghi}

Lund University

Lund, Sweden

Salam.Al-Karadaghi@biochemistry.lu.se

Chapter 137

\section{Cristina Alonso}

University of Hull

Kingston-upon-Hull, HU6 7RX, UK

Chapter 17

\section{Karl E. Anderson}

The University of Texas Medical Branch

Galveston, TX 77555-1109, USA

kanderso@utmb.edu

Chapter 152

\section{Bernie J. Anding}

Iowa State University

Ames, IA, 50010, USA

Chapter 100

\section{Edith Antunes}

Rhodes University

Grahamstown, 6139, South Africa

Chapter 34

Naoki Aratani

Kyoto University

Kyoto 606-8502, Japan

aratani@kuchem.kyoto-u.ac.jp

Chapter 1

\section{Margarida Archer}

Universidade Nova de Lisboa

2781-901 Oeiras, Portugal

Chapter 89

\section{Katsuhiko Ariga}

National Institute for Materials Science

Tsukuba, Ibaraki 305-0044, Japan

ARIGA.Katsuhiko@nims.go.jp

Chapter 81

\section{Kunio Awaga}

Nagoya University

Nagoya 464-8602, Japan

awaga@mbox.chem.nagoya-u.ac.jp

Chapter 83

\author{
Yosra M. Badiei \\ The Johns Hopkins University \\ Baltimore, Maryland 21218, USA \\ Chapter 66
}

*Full contact information for authors can be found on the title page of each chapter. 


\section{Michael N. Badminton}

University Hospital of Wales

Cardiff, CF14 4XW, UK

badmintonmn@cardiff.ac.uk

Chapter 146

\section{Ireena Bagai}

University of Michigan

Ann Arbor, MI 48109-5606, USA

Chapter 156

\section{Teodor Silviu Balaban}

Aix-Marseille University

13397 Marseille, France

ts.balaban@univ-amu.fr

Chapter 3

\author{
Alan L. Balch \\ University of California, Davis \\ Davis, CA 95616, USA \\ balch@chem.ucdavis.edu \\ Chapter 40
}

\section{David P. Ballou}

University of Michigan

Ann Arbor, MI 48109-5606, USA

Chapter 28

\section{Kylie D. Barker}

University of Maryland

Baltimore, MD 21201-1180, USA

Chapter 72

\section{Ines Batinić-Haberle}

Duke University Medical School

Durham, NC 27710, USA

ibatinic@duke.edu

Chapter 52

\section{Aurélie Bedel}

Université Bordeaux Segalen

Bordeaux, 33076, France

Chapter 154

\section{Fethi Bedioui}

Université Paris Descartes

75231 Paris cedex 05, France

fethi-bedioui@enscp.fr

Chapter 55

\section{Irina P. Beletskaya}

A.N. Frumkin Institute of Physical

Chemistry and Electrochemistry

119071 Moscow, Russian Federation

Chapter 108

\section{Ludmil Benov}

Kuwait University

13110 Safat, Kuwait

Chapter 52

Timothy C. Berto

University of Michigan

Ann Arbor, MI 48109, USA

Chapter 63

\section{Yongzhong Bian}

University of Science and Technology Beijing

Beijing 100083, China

Chapter 64

\section{Sara Bonacchi}

Università degli Studi di Bologna

40126 Bologna, Italy

Chapter 57 


\section{Christian W. von Borczyskowski}

National Technical University of Belarus

220013 Minsk, Belarus

Chapter 104

\section{Sylvia S. Bottomley}

University of Oklahoma College of Medicine

Oklahoma, OK 73104, USA

Sylvia-Bottomley@ ouhsc.edu

Chapter 144

\section{Faye Bowles}

University of California, Davis

Davis, CA 95616, USA

Chapter 40

\section{Ross W. Boyle}

University of Hull

Kingston-upon-Hull, HU6 7RX, UK

r.w.boyle@hull.ac.uk

Chapters 17, 135

\section{Ozguncem Bozkulak}

Children's Hospital Los Angeles

Los Angeles, CA 90027, USA

Chapter 22

\section{Martin Bröring}

Technische Universität Carolo-Wilhelmina zu Braunschweig

Braunschweig, Germany

m.broering@tu-bs.de

Chapter 41

\section{Markus J. Bröcker}

Yale University

New Haven

CT 06511, USA

Chapter 94

\section{Christian Brückner}

University of Connecticut

Storrs, CT 06369-3060, USA

c.bruckner@uconn.edu

Chapter 76

\author{
Donald A. Bryant \\ The Pennsylvania State University \\ University Park, PA 16802, USA \\ dab14@psu.edu \\ Chapter 96
}

\section{Christophe Bucher}

Université Joseph Fourier/CNRS

Grenoble, France

christophe.bucher@ujf-grenoble.fr

Chapter 78

\author{
Mihai Buda \\ "POLITEHNICA" University of Bucharest \\ Bucharest, Romania \\ mihai@ catedra.chfiz.pub.ro \\ Chapter 78
}

Paul W. Buehler

Center for Biologics Evaluation and Research (CBER)

Bethesda, MD 20892, USA

paul.buehler@fda.hhs.gov

Chapter 131

\section{Véronique Bulach}

Université de Strasbourg

67000 Strasbourg, France

Chapter 62

\section{Kevin Burgess}

Texas A\&M University

College Station, TX 77842, USA

burgess@tamu.edu

Chapter 37 


\section{Andrew N. Cammidge}

University of East Anglia

Norwich, NR4 7TJ, UK

a.cammidge@uea.ac.uk

Chapter 75

Christopher J. Carrell

University of Minnesota

Minneapolis, MN 55455, USA

Chapter 127

\section{Eric Carter}

University of Michigan

Ann Arbor, MI 48109-5606, USA

Chapter 156

\section{José A.S. Cavaleiro}

University of Aveiro

3810-193 Aveiro, Portugal

jcavaleiro@ua.pt

Chapter 9

\section{Isabelle Chambrier}

University of East Anglia

Norwich, NR4 7TJ, UK

i.fernandes@uea.ac.uk

Chapter 75

\section{Chi-Ming Che}

University of Hong Kong

Hong Kong, China

cmche@hku.edu

Chapter 101

\section{Caiyong Chen}

University of Maryland

Maryland 20742, USA

Chapter 67

\section{Andrei V. Cheprakov}

Moscow State University

119992, Moscow, Russia

avchep@elorg.chem.msu.ru

Chapter 58

\section{Soizic Chevance \\ Université de Rennes \\ France \\ Chapter 102}

\author{
Sung Cho \\ Yonsei University \\ Seoul, 120-747, Korea \\ Chapter 5
}

\section{Daniel P. Collins}

University of South Carolina

Columbia, SC 29208, USA

Chapter 28

\section{Michael J. Cook}

University of East Anglia

Norwich, NR4 7TJ, UK

M.Cook@uea.ac.uk

Chapter 75

\section{Maria Almira Correia \\ University of California San Francisco \\ San Francisco, CA 94158-2517, USA \\ almira.correia@ucsf.edu \\ Chapter 158}

\author{
Anne Corrigall \\ University of Cape Town \\ Rondebosch 7701, South Africa \\ Chapter 149
}


Harry A. Dailey

University of Georgia

Athens, GA 30602, USA

Chapter 123

\section{John H. Dawson}

University of South Carolina

Columbia, SC 29208, USA

dawson@mail.chem.sc.edu

Chapter 28

\section{Simon Dalgleish}

Nagoya University

Nagoya 464-8602, Japan

Chapter 83

\section{Katherine A. de Villiers}

Stellenbosch University

Stellenbosch 7602, South Africa

Chapter 133

\section{Serena DeBeer}

Cornell University

Ithaca, NY 14853, USA

Chapter 66

\section{Richard A. Decréau}

Université de Bourgogne

France

Richard.Decreau@u-bourgogne.fr

Chapter 106

\section{Philippe Delepelaire}

Université Paris Diderot - Paris 7

75252 Paris Cedex 05, France

Chapter 122

\section{Tyler G. St. Denis}

Columbia University

New York City, NY, USA

Chapter 134

\author{
Ilia G. Denisov \\ The University of Illinois \\ Urbana, IL 61801, USA \\ Denisov@illinois.edu \\ Chapter 26
}

\section{Jean-Charles Deybach \\ Université Paris Diderot \\ 75018, Paris, France \\ jean-charles.deybach@lmr.aphp.fr \\ Chapter 145}

\section{Eric Wei-Guang Diau}

National Chiao Tung University

Hsinchu, Taiwan

diau@mail.nctu.edu.tw

Chapter 142

\section{Dilek K. Dogutan}

Massachusetts Institute of Technology

Cambridge, USA

Chapter 99

\section{Charles Michael Drain}

Hunter College of The City University of New York

New York, NY 10065, USA

cdrain@hunter.cuny.edu

Chapter 15

\section{Francis D'Souza}

University of North Texas

Denton, TX 76203-5017, USA

Francis.DSouza@UNT.edu

Chapter 4

\section{Florence Duclairoir}

Institut Nanosciences et Cryogénie 38054 Grenoble cedex 9, France

Chapter 47 


\author{
Alessandro D'urso \\ Università di Catania \\ 95125 Catania, Italy \\ Chapter 59
}

\section{Jennifer L. DuBois}

Montana State University

Bozeman, MT 59717, USA

jdubois@chemistry.montana.edu

Chapters 90, 127

\section{Timothy J. Egan}

University of Cape Town

Rondebosch 7701, South Africa

Timothy.Egan@uct.ac.za

Chapter 133

\section{George H. Elder}

Cardiff University

Cardiff, CF14 4XN, UK

Chapter 146

\section{Johannes A. A. W. Elemans}

Radboud University Nijmegen

6525 AJ Nijmegen

The Netherlands

Chapter 79

\section{Juan Engel}

University of California San Francisco

San Francisco, CA 94158-2517, USA

Chapter 158

\section{Manivannan Ethirajan}

Roswell Park Cancer Institute

Buffalo, NY 14263, USA

Chapter 19
Allison J. Farrand

Vanderbilt University

Nashville, TN 37232, USA

Chapter 125

\author{
Alessandro Feis \\ University of Florence \\ I-50019 Sesto Fiorentino, Italy \\ Chapter 31
}

\section{Angela Ferrario}

Children's Hospital Los Angeles

Los Angeles, CA 90027, USA

Chapter 22

\section{Gloria C. Ferreira}

University of South Florida

Tampa, FL, 33612, USA

gferreir@health.usf.edu

Chapters 68, 119, 121

\section{Stuart J. Ferguson}

University of Oxford

Oxford, OX1 3QU, UK

stuart.ferguson@bioch.ox.ac.uk

Chapter 93

\section{Kimberly B. Fields}

University of South Florida

Tampa, FL 33620, USA

Chapters 13, 43

\section{Mark D. Fleming}

Children's Hospital and Harvard Medical School

Boston, MA 02115, USA

Chapter 144 
Antonio Fontanellas

University of Navarra

Pamplona 31008, Spain

afontanellas@unav.es

Chapter 153

\section{Nicole Frankenberg-Dinkel}

Ruhr-University Bochum

44780 Bochum, Germany

nicole.frankenberg@rub.de

Chapter 140

\section{Erica J. Fratz}

University of South Florida

Tampa, FL 33612, USA

Chapter 119

\section{Petra Fromme}

Arizona State University

Tempe, AZ 85287-1604, USA

pfromme@asu.edu

Chapter 136

\section{Raimund Fromme}

Arizona State University

Tempe, AZ 85287-1604, USA

Chapter 136

\section{Takamitsu Fukuda}

Osaka University

Toyonaka 560-0043, Japan

tfukuda@chem.sci.osaka-u.ac.jp

Chapter 42

\section{Shunichi Fukuzumi}

Osaka University

Osaka 565-0871, Japan

fukuzumi@chem.eng.osaka-u.ac.jp

Chapter 46

\section{Hiroyuki Furuta}

Kyushu University

Fukuoka 819-0395, Japan

hfuruta@cstf.kyushu-u.ac.jp

Chapter 10

\section{Kazumichi Furuyama}

Tohoku University Graduate School of Medicine

Sendai, Miyagi 980-8575, Japan

k-furuya@mail.tains.tohoku.ac.jp

Chapter 128

\section{Mary Grace I. Galinato}

University of Michigan

Ann Arbor, MI 48109, USA

Chapter 63

\section{François Moreau-Gaudry \\ Université Bordeaux Segalen \\ Bordeaux, 33076, France \\ Chapter 154}

\section{Cécile Ged}

Université Bordeaux Segalen

Bordeaux, 33076, France

Chapter 154

\section{Damiano Genovese}

Università degli Studi di Bologna

40126 Bologna, Italy

Chapter 57

\section{Peter E.M. Gibbs}

University of Rochester School of Medicine and Dentistry

Rochester, NY 14642, USA

Chapter 126 
Mallory E. Gillam

University of South Florida

Tampa, Florida FL 33612, USA

Chapter 121

\section{Jean-Paul Gisselbrecht}

Université de Strasbourg

67000 Strasbourg, France

gissel@unistra.fr

Chapter 14

\section{Francesca Giuntini}

Liverpool John Moores University

Liverpool, UK

Chapter 135

\section{David P. Goldberg}

The Johns Hopkins University

Baltimore, Maryland 21218, USA

dpg@jhu.edu

Chapter 66

\section{Charles J. Gomer}

University of Southern California

Los Angeles, CA 90027, USA

Chapter 22

\section{Lauren E. Goodrich}

University of Michigan

Ann Arbor, MI 48109, USA

Chapter 63

\section{Yulia G. Gorbunova}

A. N. Frumkin Institute of Physical Chemistry and Electrochemistry

Russian Academy of Sciences, Russia yulia@igic.ras.ru

Chapter 113

\section{Laurent Gouya}

Université Paris Diderot

75018, Paris, France

Chapter 145

\section{Bernhard Grimm}

Humboldt Universität zu Berlin

10115 Berlin, Germany

Bernhard.grimm@rz.hu-berlin.de

Chapter 138

\section{Bruno Grimm}

Friedrich-Alexander-University

Erlangen-Nuremberg

91058 Erlangen, Germany

Chapter 2

\section{Sophie Griveau}

Université Paris Descartes

75231 Paris Cedex 05, France

Chapter 55

\section{Ingo Grotjohann}

Arizona State University

Tempe, AZ 85287-1604, USA

Chapter 136

\section{Shenheng Guan}

University of California San Francisco San Francisco, CA 94158-2517, USA

Chapter 158

\section{Roger Guilard}

Université de Bourgogne

21078 Dijon Cedex, France

Roger.Guilard@u-bourgogne.fr

Chapters 49, 108 
Dirk M. Guldi

Friedrich-Alexander-University

Erlangen-Nuremberg

91058 Erlangen, Germany

dirk.guldi@chemie.uni-erlangen.de

Chapter 2

\section{Victoria L. Gunderson \\ Northwestern University \\ Evanston IL 60208-3113, USA \\ Chapter 95}

\section{Nirupama Gupta}

University of Michigan

Ann Arbor, MI 48109-5606, USA

Chapter 156

\section{Michael R. Hamblin}

Massachusetts General Hospital

Boston, MA 02114, USA

hamblin@helix.mgh.harvard.edu

Chapter 134

\section{Iqbal Hamza}

University of Maryland

College Park, MD 20742, USA

hamza@umd.edu

Chapters 67, 123

\section{Luciana Hannibal}

Cleveland Clinic

Cleveland, OH 44195, USA

hannibl@ccf.org

Chapter 157

\section{Mats Hansson}

Carlsberg Laboratory

1799 Copenhagen V, Denmark mats.hansson@carlsberglab.dk

Chapter 137

\section{Pauline Harper}

Karolinska Institutet, Karolinska

University Hospital

Stockholm, Sweden

pauline.harper@karolinska.se

Chapter 151

\section{Pierre D. Harvey \\ Université de Sherbrooke \\ Sherbrooke, J1K 2R1, Québec, Canada \\ Chapter 49}

\section{Anita Hausmann}

Friedrich-Alexander-University

Erlangen-Nuremberg

91058 Erlangen, Germany

Chapter 2

\section{Takashi Hayashi}

Osaka University

Suita 565-0871, Japan

thayashi@chem.eng.osaka-u.ac.jp

Chapter 23

\section{Dirk Heinz}

Helmholtz-Centre for Infection Research 38124 Braunschweig, Germany

Chapter 143

\section{Petra Hellwig}

Université de Strasbourg

67000 Strasbourg, France

hellwig@chimie.u-strasbg.fr

Chapter 36

\section{Carmen Herrero}

University of Barcelona

Barcelona, Spain

Chapter 147 


\section{Richard Hift}

University of KwaZulu-Natal

Durban 4041, South Africa

Chapter 149

\section{Jonathan P. Hill}

National Institute for Materials Science

Tsukuba

Ibaraki 305-0044, Japan

Jonathan.HILL@nims.go.jp

Chapter 81

\section{Yoshio Hisaeda}

Kyushu University

Fukuoka 819-0395, Japan

yhisatcm@mail.cstm.kyushu-u.ac.jp

Chapter 48

\section{Seong-Jin Hong}

Kangwon National University

Chun-Chon, 200-701, Korea

Chapter 60

\section{Stefan Hörtensteiner}

University of Zürich

CH-8008 Zürich, Switzerland

Chapter 139

\section{Mir Wais Hosseini}

Université de Strasbourg

67000 Strasbourg, France

hosseini@chimie.u-strasbg.fr

Chapter 62

\section{Barry D. Howes}

University of Florence

I-50019 Sesto Fiorentino, Italy

Chapter 31

\section{Ying-Ying Huang}

Massachusetts General Hospital

Boston, MA 02114, USA

Chapter 134

\section{Gregory A. Hunter}

University of South Florida

Tampa, FL, 33612, USA

Chapters 68, 121

\section{Jotaro Igarashi}

Tohoku University

Katahira, Sendai 980-8577, Japan

Chapter 73

\section{Akira Ikezaki \\ Toho University \\ Tokyo 143-8540, Japan \\ Chapter 32}

\section{Shinsuke Ishihara}

National Institute for Materials Science

Tsukuba, Ibaraki 305-0044, Japan

Chapter 81

\section{Osamu Ito}

Tohoku University

Sendai, 981-3215, Japan

Chapter 4

\section{Hisashi Ito}

Hokkaido University

Sapporo 060-0819, Japan

Chapter 98

\section{Anabella Ivancich}

Centre Nationale de la Recherche

Scientifique

F-91191 Gif-sur-Yvette, France

Chapter 31 


\section{Hiroshi Imahori}

Kyoto University

Kyoto 615-8510, Japan

imahori@ scl.kyoto-u.ac.jp

Chapter 80

\section{Eileen K. Jaffe}

Fox Chase Cancer Center, Temple Health

Philadelphia, PA 19111, USA

eileen.jaffe@fccc.edu

Chapter 120

\section{Dieter Jahn}

Technische Universität Braunschweig

D-38106 Braunschweig, Germany

d.jahn@tu-bs.de

Chapters 70, 94, 143

\section{Martina Jahn}

Technische Universität Braunschweig

D-38106 Braunschweig, Germany

Chapters 70, 143

\section{Christophe Jeandon}

Université de Strasbourg

67000 Strasbourg, France

cjeandon@unistra.fr

Chapter 14

\section{Yiping Jia}

Center for Biologics Evaluation and Research (CBER)

Bethesda, MD 20892, USA

Chapter 131

\section{Jianzhuang Jiang}

University of Science and Technology

$$
\text { Beijing }
$$

Beijing 100083, China

jianzhuang@ustb.edu.cn

Chapter 64

\section{Riccardo Juris}

Università degli Studi di Bologna

40126 Bologna, Italy

Chapter 57

\author{
Huangxian Ju \\ Nanjing University \\ Nanjing 210093, China \\ hxju@nju.edu.cn \\ Chapter 82
}

\section{Norbert Jux}

Universität Erlangen-Nürnberg

91054 Erlangen, Germany

norbert.jux@chemie.uni-erlanger.de

Chapters 20, 112

\section{Axel Kahnt \\ Friedrich-Alexander-University \\ Erlangen-Nuremberg \\ 91058 Erlangen, Germany}

Chapter 2

\section{Toshiaki Kamachi}

Tokyo Institute of Technology

Yokohama 226-8501, Japan

Chapter 56

\section{Yoriko Kanai}

Shinshu University

Ueda, 386-8567, Japan

Chapter 53

\section{Zoubida Karim}

Université Paris Diderot

75018, Paris, France

Chapter 145 


\section{Anna-Lena Kaufholz}

Technische Universität Braunschweig

D-38106 Braunschweig, Germany

Chapter 143

\section{James M. Kelly}

University of Cambridge

Cambridge, CB2 1EW, UK

Chapter 115

\section{David Kessel}

Wayne State University School of Medicine

Detroit, MI 48201, USA

dhkessel@med.wayne.edu

Chapter 21

\section{Dongho Kim}

Yonsei University

Seoul, 120-747, Korea

dongho@yonsei.ac.kr

Chapters 5, 6

\section{Kil Suk Kim}

Yonsei University

Seoul, 120-747, Korea

Chapter 6

\section{Kenichi Kitanishi}

Tohoku University

Sendai 980-8577, Japan

Chapter 73

\section{Nagao Kobayashi}

Tohoku University

Sendai 980-8578, Japan

nagaok@m.tohoku.ac.jp

Chapters 33, 42, 109, 110

\section{Koichi Kobayashi}

RIKEN Institute, Japan

kouity@pcs.riken.jp

Chapter 97

\section{Mark J. Koury}

Vanderbilt University

Nashville, TN 37212, USA

Chapter 129

\section{Bernhard Kräutler}

University of Innsbruck

A-6020 Innsbruck, Austria

Bernard.Kraeutler@uibk.ac.at

Chapters 117, 139

\section{Christopher Kupitz}

Arizona State University

Tempe, AZ 85287-1604, USA

Chapter 136

\section{Kei Kurotobi}

Kyoto University

Kyoto 615-8510, Japan

Chapter 80

\section{Michio Kunieda}

Ritsumeikan University

Shiga 525-8577, Japan

Chapter 51

\section{Daniela Lalli}

University of Florence

I-50019 Sesto Fiorentino, Italy

Chapter 160

\section{Randy W. Larsen}

University of South Florida

Florida FL 33620, USA

rwlarsen@usf.edu

Chapter 161 
Timothy D. Lash

Illinois State University

Normal, Illinois 61790-4160, USA

tdlash@ilstu.edu

Chapter 74

\section{Lechosław Latos-Grażyński}

University of Wrockaw

Wrockaw 50 383, Poland

llg@wchuwr.pl

Chapter 8

\section{Janet T. F. Lau}

The Chinese University of Hong Kong

Hong Kong, China

Chapter 84

\section{Rosaria Lauceri}

Università di Catania

95125 Catania, Italy

Chapter 59

\section{Andrew D. Lawrence}

University of Kent

Canterbury, Kent, CT2 7NZ, UK

A.Lawrence-219@kent.ac.uk

Chapter 115

\section{Sarah H. Lawrence}

Fox Chase Cancer Center, Temple Health

Philadelphia, PA 19111, USA

Chapter 120

\section{Gunhild Layer}

Technische Universität Braunschweig

D-38106 Braunschweig, Germany

g.layer@tu-braunschweig.de

Chapters 70, 88, 143

\section{Chang-Hee Lee}

Kangwon National University

Chun-Chon, 200-701, Korea

Chapter 60

\section{Pannee Leeladee}

The Johns Hopkins University

Baltimore, Maryland 21218, USA

Chapter 66

\section{Finian J. Leeper}

University of Cambridge

Cambridge, CB2 1EW, UK

Chapter 115

\author{
Nicolai Lehnert \\ University of Michigan \\ Ann Arbor, MI 48109, USA \\ lehnert@umich.edu \\ Chapter 63
}

\section{Christopher M. Lemon}

Massachusetts Institute of Technology

Cambridge, USA

clemon@mit.edu

Chapter 99

\author{
Jianping Lei \\ Nanjing University \\ Nanjing 210093, China \\ jpl@nju.edu.cn \\ Chapter 82
}

\section{Dennis Leupold}

University of Bremen

D-28359 Bremen, Germany

Chapter 118 


\section{Genxi Li}

Nanjing University

Nanjing 210093, PR China

genxili@nju.edu.cn

Chapter 27

\section{Lu-Lin Li}

National Chiao Tung University

Hsinchu, Taiwan

Chapter 142

\section{Mingxiang Liao}

University of California San Francisco

San Francisco, CA 94158-2517, USA

Chapter 158

\section{Jong Min Lim}

Yonsei University

Seoul, 120-747, Korea

Chapter 6

\section{Jonathan S. Lindsey}

North Carolina State University

Raleigh, USA

jlindsey@ncsu.edu

Chapter 107

\section{Yi Liu}

University of California San Francisco San Francisco, CA 94158-2517, USA

Chapter 158

\section{Zhenfeng Liu}

The Pennsylvania State University

University Park

PA 16802, USA

Chapter 96

\section{Pui-Chi Lo}

The Chinese University of Hong Kong

Hong Kong, China

pclo@cuhk.edu.hk

Chapter 84

\section{Vanessa Kar-Yan Lo \\ University of Hong Kong \\ Hong Kong, China \\ Chapter 101}

\section{Susana A. Lobo \\ Universidade Nova de Lisboa \\ 2781-901 Oeiras, Portugal \\ Chapter 89}

\author{
Aurore Loudet \\ Texas A\&M University \\ College Station, TX 77842, USA \\ Chapter 37
}

\author{
Ricardo O. Louro \\ Universidade Nova de Lisboa \\ 2781-901 Oeiras, Portugal \\ Chapter 89
}

\section{Gudrun S. Lukat-Rodgers}

North Dakota State University

Fargo, ND 58102, USA

Gudrun.Lukat-rodgers@ndsu.edu

Chapter 162

\section{Evgeny A. Lukyanets}

Organic Intermediates and Dyes Institute Moscow, 123995, Russia

rmeluk@niopik.ru

Chapter 11 


\section{Marian Luna}

Children's Hospital Los Angeles

Los Angeles, CA 90027, USA

Chapter 22

\section{Joakim Lundqvist}

Carlsberg Laboratory

1799 Copenhagen V, Denmark

Chapter 137

\section{Stuart A. MacGowan}

Trinity College Dublin

Dublin 2, Ireland

Chapter 61

\section{John Mack}

Rhodes University

Grahamstown 6139, South Africa

mack@uwo.ca

Chapter 109

\section{Hiromitsu Maeda}

Ritsumeikan University

Kusatsu 525-8577, Japan

maedahir@ph.ritsumei.ac.jp

Chapter 38

\section{Mahin D. Maines}

University of Rochester School of Medicine and Dentistry

Rochester, NY 14642, USA

Mahin_Maines@urmc.rochester.edu

Chapter 126

\section{Fabrizio Mancin}

Università degli Studi di Bologna 40126 Bologna, Italy

Chapter 57
Jean-Claude Marchon

Institut Nanosciences et Cryogénie 38054 Grenoble Cedex 9, France jean-claude.marchon@cea.fr Chapter 47

\author{
Michael A. Marletta \\ University of California, Berkeley \\ Berkeley, USA \\ marletta@berkeley.edu \\ Chapter 69
}

Kathleen E. Martin

University of New Mexico

New Mexico NM 87106, USA

Chapter 141

\section{Victoria Martínez-Díaz \\ Universidad Autónoma de Madrid 28049-Madrid, Spain \\ Chapter 45}

\section{Alexander G. Martynov \\ N.S. Kurnakov Institute of General and Inorganic Chemistry \\ Russian Academy of Sciences, Russia \\ Chapter 113}

\section{Tatsuru Masuda}

The University of Tokyo

Tokyo 153-8902, Japan

ctmasuda@mail.ecc.u-tokyo.ac.jp

Chapter 97

\section{Pedro M. Matias}

Universidade Nova de Lisboa

2781-901 Oeiras, Portugal

matias@itqb.unl.pt

Chapter 89 


\section{A. Grant Mauk}

University of British Columbia, Vancouver

British Columbia V6T 1 Z3 Canada

mauk@exchange.ubc.ca

Chapter 92

\section{Paul Le Maux}

Université de Rennes

France

Chapter 102

\section{Jeffery A. Mayfield}

University of Notre Dame

Notre Dame, Indiana 46556, USA

Chapter 90

\section{Amanda J. McGown}

The Johns Hopkins University

Baltimore, Maryland 21218, USA

Chapter 66

\section{Craig J. Medforth}

University of New Mexico

Albuquerque, NM 87106, USA

Chapters 50, 141

\section{Takashi Megumi}

Shinshu University

Ueda, 386-8567, Japan

Chapter 53

\section{Ignacio Melero}

University of Navarra

Pamplona 31008, Spain

Chapter 153

\section{Peter Meissner}

University of Cape Town

Rondebosch 7701, South Africa

Peter.Meissner@uct.ac.za

Chapter 149

\section{Frederic Melin}

Université de Strasbourg

67000 Strasbourg, France

Chapter 36

\section{Oscar Millet}

Parque Tecnológico de Bizkaia

Derio, Spain

Chapter 147

\author{
Elisabeth I. Minder \\ Stadtspital Triemli \\ Zürich, Switzerland \\ elisabeth.minder@ triemli.stzh.ch \\ Chapter 150
}

\section{Andrey Mironov}

Lomonosov Moscow State University of Fine Chemical Technologies

Moscow, Russia

mironov@mitht.ru

Chapter 85

\section{Marco Montalti}

Università degli Studi di Bologna

40126 Bologna, Italy

Chapter 57

\section{Donato Monti}

University of Rome "Tor Vergata"

00133 Rome, Italy

Chapter 54

\section{Franz-Peter Montforts}

University of Bremen

D-28359 Bremen, Germany

mont@uni-bremen.de

Chapter 118 


\section{Jürgen Moser}

Technische Universität Braunschweig

D-38106 Braunschweig, Germany

j.moser@tu-bs.de

Chapter 94

\section{Arun Nagaraj}

Indian Institute of Science

Bangalore-560012, India

Chapter 132

\section{Shingo Nagano}

Tottori University

Tottori 680-8552, Japan

Chapter 25

\section{Mikio Nakamura}

Toho University

Tokyo 143-8540, Japan

mnakamu@med.toho-u.ac.jp

Chapter 32

\section{Daniel G. Nocera}

Massachusetts Institute of Technology

Cambridge, USA

nocera@mit.edu

Chapter 99

\section{Wonwoo Nam}

Ewha Womans University

Seoul 120-750, South Korea

wwnam@ewha.ac.kr

Chapter 44

\section{Sara Nardis}

University of Rome "Tor Vergata"

00133 Rome, Italy

Chapter 54
Corrado Di Natale

University of Rome "Tor Vergata"

00133 Rome, Italy

Chapter 54

\section{Maura J. O’Neill}

University of Maryland

Baltimore, MD 21201-1180, USA

Chapter 124

\section{Victor N. Nemykin}

University of Minnesota Duluth

Duluth, MN 55812, USA

vnemykin@d.umn.edu

Chapter 11

\author{
Maria G.P.M.S. Neves \\ University of Aveiro \\ 3810-193 Aveiro, Portugal \\ Chapter 9
}

\section{Huy-Danh Nguyen-Tran}

Shinshu University

Ueda, 386-8567, Japan

Chapter 53

\section{Dennis K. P. Ng}

The Chinese University of Hong Kong Hong Kong, China

dkpn@cuhk.edu.hk

Chapter 84

\section{Roeland J. M. Nolte}

Radboud University Nijmegen

6525 AJ Nijmegen

The Netherlands

r.nolte@ science.ru.nl

Chapter 79 


\section{Tebello Nyokong}

Rhodes University

Grahamstown, 6139, South Africa

t.nyokong@ru.ac.za

Chapters 34, 114

\section{Junichi Ogikubo}

University of Connecticut, Storrs

CT 06369-3060, USA

Chapter 76

\section{Yoshiki Ohgo}

Toho University

Tokyo 143-8540, Japan

Chapter 32

\section{Kazuchika Ohta}

Shinshu University

Ueda, 386-8567, Japan

ko52517@shinshu-u-ac.jp

Chapter 53

\section{Tetsuo Okujima}

Ehime University

Matsuyama 790-8577, Japan

Chapter 7

\section{Ichiro Okura}

Tokyo Institute of Technology

Yokohama 226-8501, Japan

iokura@bio.titech.ac.jp

Chapter 56

\section{Noboru Ono}

Ehime University

Matsuyama 790-8577, Japan

ononbr@dpc.ehime-u.ac.jp

Chapter 7
Paul R. Ortiz de Montellano

University of California

San Francisco, CA 94158-2517, USA

ortiz@cgl.ucsf.edu

Chapter 155

\section{Atsuhiro Osuka \\ Kyoto University \\ Kyoto 606-8502, Japan \\ osuka@kuchem.kyoto-u.ac.jp \\ Chapter 1}

\section{Martina Osmers}

University of Bremen

D-28359 Bremen, Germany

Chapter 118

\section{Zhongping Ou}

Jiangsu University

Zhenjiang 212013, China

Chapter 64

\author{
Kristina E. Overkamp \\ Ruhr-University Bochum \\ 44780 Bochum, Germany \\ Chapter 140
}

\section{Govindarajan Padmanaban}

Indian Institute of Science

Bangalore-560012, India

geepee@biochem.iisc.ernet.in

Chapter 132

\author{
Ravindra K. Pandey \\ Roswell Park Cancer Institute \\ Buffalo, NY 14263, USA \\ ravindra.pandey@ roswellpark.org \\ Chapter 19
}




\section{Roberto Paolesse}

University of Rome "Tor Vergata"

00133 Rome, Italy

roberto.paolesse@uniroma2.it

Chapter 54

\section{Nayan J. Patel}

Roswell Park Cancer Institute

Buffalo, NY 14263, USA

Chapter 19

\section{Barry H. Paw}

Harvard Medical School

Boston, MA 02115, USA

bpaw@rics.bwh.harvard.edu

Chapter 130

\section{Miłosz Pawlicki}

University of Wrocław

Wrocław 50 383, Poland

Chapter 8

Inês A. C. Pereira

Universidade Nova de Lisboa

2781-901 Oeiras, Portugal

Chapter 89

\section{Claudia P. Pereira}

Center for Biologics Evaluation and Research (CBER)

Bethesda, MD 20892, USA

Chapter 131

\section{John D. Phillips}

University of Utah School of Medicine Salt Lake City, Utah 84132, USA

john.phillips@hsc.utah.edu

Chapters 91, 148

\section{Prem Ponka}

Lady Davis Institute for Medical Research Montréal, QC H3T 1E2, Canada

prem.ponka@mcgill.ca

Chapter 129

\section{Robert J. Porra \\ CSIRO-Plant Industry \\ Canberra, ACT 2601, Australia \\ Chapter 103}

\author{
Thomas L. Poulos \\ University of California Irvine \\ Irvine, California 92697-3900, USA \\ poulos@uci.edu \\ Chapter 87
}

\section{Luca Prodi}

Università degli Studi di Bologna

40126 Bologna, Italy

luca.prodi@unibo.it

Chapter 57

\section{Katharine A. Prokop \\ The Johns Hopkins University \\ Baltimore, Maryland 21218, USA \\ Chapter 66}

\section{Barbara Puffer}

University of Innsbruck

A-6020 Innsbruck, Austria

Barbara.Puffer@uibk.ac.at

Chapter 117

\section{Roberto Purrello}

Università di Catania

95125 Catania, Italy

r.purrello@unict.it

Chapter 59 


\section{Hervé Puy}

Université Paris Diderot

75018, Paris, France

herve.puy@bch.aphp.fr

Chapter 145

\section{Stephen W. Ragsdale}

University of Michigan

Ann Arbor, MI 48109-0606, USA

sragsdal@umich.edu

Chapters 86, 156

\section{Enrico Rampazzo}

Università degli Studi di Bologna

40126 Bologna, Italy

Chapter 57

\section{Pundi N. Rangarajan}

Indian Institute of Science

Bangalore-560012, India

Chapter 132

\section{Júlio S. Rebouças}

Universidade Federal da Paraíba

João Pessoa, PB 58051-970, Brazil

Chapter 52

\section{Giampaolo Ricciardi}

Università della Basilicata

85100 Potenza, Italy

Chapter 105

\section{Emmanuel Richard}

Université Bordeaux Segalen

Bordeaux, 33076, France

Chapter 154

\section{Sébastien Richeter}

Université Montpellier 2

34095 Montpellier Cedex 5, France sebastien.richeter@univ-montp2.fr

Chapter 14

\author{
Mario Rivera \\ University of Kansas \\ Lawrence, KS 66047, USA \\ mrivera@ku.edu \\ Chapter 159
}

\section{Beate Röder}

Humboldt-Universität zu Berlin

12489 Berlin, Germany

roeder@physik.hu-berlin.de

Chapter 20

\section{Vladimir Roznyatovskiy}

The University of Texas at Austin Austin, Texas, 78712, USA

Chapter 60

\section{Célia V. Romão}

Universidade Nova de Lisboa

2781-901 Oeiras, Portugal

Chapter 89

\section{Kenton R. Rodgers}

North Dakota State University

Fargo, ND 58102, USA

Kent.Rodgers@ndsu.edu

Chapter 162

\section{Angela Rosa}

Università della Basilicata

85100 Potenza, Italy

angela.rosa@unibas.it

Chapter 105 


\author{
Alan E. Rowan \\ Radboud University Nijmegen \\ 6525 AJ Nijmegen \\ The Netherlands \\ A.Rowan@science.ru.nl \\ Chapter 79 \\ Natalie Rucker \\ Children's Hospital Los Angeles \\ Los Angeles, CA 90027, USA \\ Chapter 22
}

Alexander B. Rudine
Portland State University
Portland OR 97207-0751, USA
Chapter 80

Joshua V. Ruppel

University of South Florida

Tampa, FL 33620, USA

Chapters 13, 43

\section{Romain Ruppert}

Université de Strasbourg

67000 Strasbourg, France

rruppert@unistra.fr

Chapter 14

\section{Aoife Ryan}

Trinity College Dublin

Dublin 2, Ireland

Chapter 12

\section{Annabel Salinas}

Humboldt Universität zu Berlin

10115 Berlin, Germany

Chapter 138

\section{Lalith Samankumara}

University of Connecticut, Storrs

CT 06369-3060, USA

Chapter 76

\section{Lígia M. Saraiva}

Universidade Nova de Lisboa

2781-901 Oeiras, Portugal

Chapter 89

\section{Hugo Scheer}

Universität München

D-80638 Munchen, Germany

H.Scheer@biologie.uni-muenchen.de

Chapter 103

\author{
W. Robert Scheidt \\ University of Notre Dame \\ Notre Dame, IN 46556-5670, USA \\ scheidt1@nd.edu \\ Chapter 111
}

\section{Derck Schlettwein}

Justus-Liebig-University Giessen

D-35392 Giessen, Germany

Derck.Schlettwein@ap.physik.uni-giessen.de

Chapter 114

\section{Xiaoye Schneider-Yin}

Stadtspital Triemli

Zürich, Switzerland

Xiaoye.Schneider@triemli.zuerich.ch

Chapter 150

\section{Wolfgang Seitz}

Friedrich-Alexander-University

Erlangen-Nuremberg

91058 Erlangen, Germany

Chapter 2 


\section{Mathias O. Senge}

Trinity College Dublin

Dublin 2, Ireland

sengem@tcd.ie

Chapters 12, 61

Natalia N. Sergeeva

Trinity College Dublin

Dublin 2, Ireland

Chapter 12

Jonathan L. Sessler

The University of Texas at Austin

Austin, Texas, 78712, USA

sessler@mail.utexas.edu

Chapter 60

\section{Scott Severance}

University of Maryland

Maryland 20742, USA

Chapter 67

\section{Dhvanit I. Shah}

Harvard Medical School

Boston MA 02115, USA

Chapter 130

\author{
Alex D. Sheftel \\ University of Ottawa Heart Institute \\ ON K1Y 4W7, Canada \\ Chapter 129
}

\section{John A. Shelnutt}

University of Georgia

Athens GA 30602, USA

jasheln@unm.edu

Chapters 50, 141
Zhen Shen

Nanjing University

Nanjing 210093, China

zshen@nju.edu.cn

Chapter 109

\section{Hisashi Shimakoshi \\ Kyushu University \\ Fukuoka 819-0395, Japan \\ Chapter 48}

\section{Toru Shimizu}

Tohoku University

Sendai 980-8577, Japan

shimizu@ agen.tohoku.ac.jp

Chapter 73

\section{Jae-Yoon Shin}

Yonsei University

Seoul, 120-747, Korea

Chapter 6

\section{Yoshitsugu Shiro}

Harima Institute

Hyogo 679-5148, Japan

yshiro@ riken.jp

Chapters 24, 25

\section{Martha Sibrian-Vazquez}

Portland State University

Portland, OR 97201, USA

Chapters 18, 135

\section{Gérard Simonneaux}

Campus de Beaulieu

Université de Rennes, France

gerard.simonneaux@univ-rennes1.fr

Chapter 102 


\section{Ashwani K. Singal}

Mayo Clinic

Rochester, MN55905, USA

aksingal@utmb.edu

Chapter 148

\section{Sunaina Singh}

Hunter College of The City University of New York

New York, NY 10065, USA

Chapter 15

Nick Sirijovski

Lund University

Lund, Sweden

Chapter 137

Eric P. Skaar

Vanderbilt University

Nashville, TN 37232, USA

eric.skaar@Vanderbilt.Edu

Chapter 125

\section{Dariusz A. Sliwa}

University of Michigan

Ann Arbor, MI 48109-0606, USA

Chapter 86

\section{Stephen G. Sligar}

The University of Illinois

Urbana, IL 61801, USA

s-sligar@uiuc.edu

Chapter 26

\section{Ann Smith}

University of Missouri

Kansas City, MO 64110, USA

SmithAn@umkc.edu

Chapters 71, 163

\section{Giulietta Smulevich}

University of Florence

I-50019 Sesto Fiorentino, Italy

giulietta.smulevich@unifi.it

Chapter 31

\author{
Nicole L. Snyder \\ Hamilton College \\ Clinton, NY 13323, USA \\ Chapters 13, 43
}

\section{Lydia Sosa-Vargas \\ University of East Anglia \\ Norwich, NR4 7TJ, UK \\ Chapter 75}

\section{Fabian Spänig}

Friedrich-Alexander-University

Erlangen-Nuremberg

91058 Erlangen, Germany

Chapter 2

\section{Ivan Spasojević}

Duke University Medical School Durham NC 27710, USA

Chapter 52

\section{Andrea Morris Spencer}

University of Michigan

Ann Arbor, MI 48109-5606, USA

Chapter 156

\section{Tatyana Spolitak}

University of Michigan

Ann Arbor, MI 48109-5606, USA

Chapter 28 
Hassan Srour

Campus de Beaulieu

Université de Rennes

1, 35042 Rennes, France

Chapter 102

\section{Christine Stern}

Université de Bourgogne

21078 Dijon Cedex, France

Christine.Stern@u-bourgogne.fr

Chapters 49, 108

\section{Julie M. Stevens}

University of Oxford

Oxford, OX1 3QU, UK

julie.stevens@bioch.ox.ac.uk

Chapter 93

\section{Martin J. Stillman}

The University of Western Ontario

London, Ontario N6A 5B7, Canada

stillman@uwo.ca

Chapter 65

\section{Bosko M. Stojanovski}

University of South Florida

Tampa, FL 33612, USA

Chapter 119

\section{Dennis J. Stuehr}

Cleveland Clinic

Cleveland, OH 44195, USA

stuehrd@ccf.org

Chapter 157

\section{Hiroshi Sugimoto}

Harima Institute

Hyogo 679-5148, Japan

Chapter 24
Yasufumi Takagi

Shinshu University

Ueda, 386-8567, Japan

Chapter 53

\section{Atsushi Takabayashi}

Hokkaido University

Sapporo 060-0819, Japan

Chapter 98

\section{Shin-ichi J. Takayama}

University of British Columbia

Vancouver

British Columbia V6T 1 Z3 Canada

Chapter 92

\section{Osamu Takikawa}

National Center for Geriatrics and Gerontology

Aichi 474-8522, Japan

Chapter 24

\section{Hitoshi Tamiaki}

Ritsumeikan University

Shiga 525-8577, Japan

tamiaki@ph.ritsumei.ac.jp

Chapter 51

\section{Ayumi Tanaka}

Hokkaido University

Sapporo 060-0819, Japan

ayumi@pop.lowtem.hokudai.ac.jp

Chapter 98

\section{Ryouichi Tanaka}

Hokkaido University

Sapporo 060-0819, Japan

rtanaka@lowtem.hokudai.ac.jp

Chapter 98 


\section{Masahiko Taniguchi}

North Carolina State University

Raleigh, NC 27695, USA

Chapter 107

\section{Lisa Tauchi}

Shinshu University

3-15-1 Tokida, Ueda, 386-8567, Japan

Chapter 53

Miguel Teixeira

Universidade Nova de Lisboa

2781-901 Oeiras, Portugal

Chapter 89

\author{
Alison Thompson \\ Dalhousie University \\ Halifax, Nova Scotia, Canada \\ alison.thompson@dal.ca \\ Chapter 39
}

\section{Yongming Tian}

New Mexico Institute of Mining and Technology

New Mexico, NM 87801, USA

Chapter 141

\section{Jordi To-Figueras}

University of Barcelona

Barcelona, Spain

JTO@clinic.ub.es

Chapter 147

\section{Motoki Toganoh}

Kyushu University

Fukuoka 819-0395, Japan

Chapter 10
Augusto C. Tomé

University of Aveiro

3810-193 Aveiro, Portugal

Chapter 9

\section{Tomas Torres}

Universidad Autónoma de Madrid

28049-Madrid, Spain

tomas.torres@uam.es

Chapter 45

\section{Aslan Yu. Tsivadze}

N.S. Kurnakov Institute of General and Inorganic Chemistry

Russian Academy of Sciences, Russia

Chapter 113

\section{Paola Turano}

University of Florence

I-50019 Sesto Fiorentino, Italy

turano@cerm.unifi.it

Chapters 30, 160

\section{Vladimir S. Tyurin}

A.N. Frumkin Institute of Physical

Chemistry and Electrochemistry

119071 Moscow, Russian Federation

Chapter 108

\section{Md. Imam Uddin}

Dalhousie University

Halifax, Nova Scotia, Canada

Chapter 39

\section{Alexei Uglov}

Université de Bourgogne

21078 Dijon Cedex, France

Chapter 108 
Johan E. van Lier

Université de Sherbrooke

Sherbrooke, Québec, Canada

Johannes.Van.Lier@USherbrooke.ca

Chapter 16

\author{
Nico Veling \\ Radboud University Nijmegen \\ 6525 AJ Nijmegen \\ The Netherlands \\ Chapter 79
}

\section{Hubert de Verneuil}

Université Bordeaux Segalen

Bordeaux, 33076, France

Hubert.De.Verneuil@u-bordeaux2.fr

Chapter 154

\section{Maria da Graça H. Vicente}

Louisiana State University

Baton Rouge, LA 70803, USA

vicente@1su.edu

Chapters 18, 135

\section{Sam P. de Visser}

The University of Manchester

Manchester M1 7DN, UK

sam.devisser@manchester.ac.uk

Chapter 44

\section{Staffan Wahlin}

Karolinska Institutet, Karolinska University Hospital

Stockholm, Sweden

staffan.wahlin@karolinska.se

Chapter 151

\section{F. Ann Walker}

University of Arizona

Tucson, AZ 85721-0041, USA

awalker@email.arizona.edu

Chapter 29

\section{Michael G. Walter \\ University of North Carolina at Charlotte \\ Charlotte, NC 28223, USA \\ Chapter 80}

\section{Jacek Waluk}

Polish Academy of Sciences

01-224 Warsaw, Poland

waluk@ichf.edu.pl

Chapter 35

Carl C. Wamser

Portland State University

Portland, OR 97207-0751, USA

wamserc@pdx.edu

Chapter 80

\section{Cécile Wandersman}

Institut Pasteur

75724 Paris Cedex 15, France

cwander@pasteur.fr

Chapter 122

\section{YongQiang Wang}

University of California San Francisco San Francisco, CA 94158-2517, USA

Chapter 158

\section{Martin J. Warren}

University of Kent

Canterbury, Kent, CT2 7NZ, UK

m.j.warren@kent.ac.uk

Chapters 88, 115 
Michael R. Wasielewski

Northwestern University

Evanston IL 60208-3113, USA

m-wasielewski@northwestern.edu

Chapter 95

\section{Emily E. Weinert}

University of California, Berkeley

Berkeley, CA, USA

Chapter 69

\section{Sharon D. Whatley}

University Hospital of Wales

Cardiff, CF14 4XW, UK

Chapter 146

\section{Angela Wilks}

University of Maryland

Baltimore, MD 21201-1180, USA

awilks@ rx.umaryland.edu

Chapters 72, 124

\section{Carrie M. Wilmot}

University of Minnesota

Minneapolis, MN 55455, USA

Chapter 127

\section{Sam Wong}

Children's Hospital Los Angeles

Los Angeles, CA 90027, USA

Chapter 22

\section{Keith Woo}

Iowa State University

Ames, IA, 50010, USA

kwoo@iastate.edu

Chapter 100
Tabitha E. Wood

Dalhousie University

Halifax, Nova Scotia, Canada

Chapter 39

\section{Frank Xu}

Children's Hospital Los Angeles

Los Angeles, CA 90027, USA

Chapter 22

\section{Hiroko Yamada}

Ehime University

Matsuyama 790-8577, Japan

Chapter 7

\section{Masayuki Yamamoto}

Tohoku University Graduate School of Medicine

Sendai, Miyagi 980-8575, Japan

masiyamamoto@med.tohoku.ac.jp

Chapter 128

\section{Jaesung Yang}

Yonsei University

Seoul, 120-747, Korea

Chapter 5

\section{Hyejin Yoo}

Yonsei University

Seoul, 120-747, Korea

Chapter 5

\section{Min-Chul Yoon}

Yonsei University

Seoul, 120-747, Korea

Chapter 6 


\section{Zin Seok Yoon}

Yonsei University

Seoul, 120-747, Korea

Chapter 5

\section{Nelsi Zaccheroni}

Università degli Studi di Bologna 40126 Bologna, Italy

Chapter 57

\section{Eduard I. Zenkevich}

National Technical University of Belarus

220013 Minsk, Belarus

zenkev@ tut.by

Chapter 104

\section{Kai-Hong Zhao}

Huazhong Agricultural University

Wuhan 430070, China

Chapter 103

\section{Peter Zhang}

University of South Florida

Tampa, FL 33620, USA

pzhang@cas.usf.edu

Chapters 13, 43

\section{Yuexing Zhang}

University of Science and Technology Beijing

Beijing 100083, China

Chapter 64

\section{Felix Zelder}

University of Zürich

CH-8057 Zürich, Switzerland zelder@aci.uzh.ch

Chapter 116

\section{Yuzhen Zhou}

University of Michigan

Ann Arbor, MI 48109-0606, USA

Chapter 86

\section{Cong-Ying Zhou}

University of Hong Kong

Hong Kong, China

Chapter 101

\author{
Christopher J. Ziegler \\ University of Akron \\ Akron OH 44325-3501 USA \\ ziegler@uakron.edu \\ Chapter 77
}




\section{Contents of Volumes 1-30}

\section{Volume 1 Supramolecular Chemistry}

1. Synthetic Strategies Toward Multiporphyrinic Architectures Naoki Aratani and Atsuhiro Osuka

2. Charge Transfer Between Porphyrins/Phthalocyanines and Carbon Nanostructures

Bruno Grimm, Anita Hausmann, Axel Kahnt, Wolfgang Seitz, Fabian Spänig and Dirk M. Guldi

3. Self-Assembling Porphyrins and Chlorins as Synthetic Mimics of the Chlorosomal Bacteriochlorophylls

Teodor Silviu Balaban

4. Tetrapyrrole-Nanocarbon Hybrids: Self-Assembly and Photoinduced Electron Transfer Francis D'Souza and Osamu Ito

5. Photophysical Properties of Various Directly Linked Porphyrin Arrays Zin Seok Yoon, Jaesung Yang, Hyejin Yoo, Sung Cho and Dongho Kim

6. Photophysics and Photochemistry of Various Expanded Porphyrins Jong Min Lim, Min-Chul Yoon, Kil Suk Kim, Jae-Yoon Shin and Dongho Kim

\section{Volume 2 Synthesis and Coordination Chemistry}

7. Synthesis of Porphyrins Fused with Aromatic Rings Noboru Ono, Hiroko Yamada and Tetsuo Okujima

8. Carbaporphyrinoids - Synthesis and Coordination Properties Miłosz Pawlicki and Lechosław Latos-Grażyński

9. meso-Tetraarylporphyrin Derivatives: New Synthetic Methodologies José A.S. Cavaleiro, Augusto C. Tomé and Maria G.P.M.S. Neves

10. Synthesis and Metal Coordination of N-Confused and N-Fused Porphyrinoids Motoki Toganoh and Hiroyuki Furuta 


\section{Volume 3 Synthetic Methodology}

11. The Key Role of Peripheral Substituents in the Chemistry of Phthalocyanines

Victor N. Nemykin and Evgeny A. Lukyanets

12. Organometallic C-C Coupling Reactions for Porphyrins Natalia N. Sergeeva, Mathias O. Senge and Aoife Ryan

13. Porphyrin Functionalization via Palladium-Catalyzed Carbon-Heteroatom Cross-Coupling Reactions

Kimberly B. Fields, Joshua V. Ruppel, Nicole L. Snyder and X. Peter Zhang

14. Peripherally Metalated Porphyrin Derivatives: Synthetic Approaches and Properties

Sébastien Richeter, Christophe Jeandon, Jean-Paul Gisselbrecht and Romain Ruppert

15. Combinatorial Libraries of Porphyrins: Chemistry and Applications Charles Michael Drain and Sunaina Singh

\section{Volume 4 Phototherapy, Radioimmunotherapy and Imaging}

16. Porphyrins and Phthalocyanines as Photosensitizers and Radiosensitizers Hasrat Ali and Johan E. van Lier

17. Bioconjugates of Porphyrins and Related Molecules for Photodynamic Therapy

Cristina Alonso and Ross W. Boyle

18. Syntheses of Boronated Porphyrins and Their Application in BNCT Maria da Graça H. Vicente and Martha Sibrian-Vazquez

19. Porphyrin-Based Multifunctional Agents for Tumor-Imaging and Photodynamic Therapy (PDT)

Manivannan Ethirajan, Nayan J. Patel and Ravindra K. Pandey

20. Targeting Strategies for Tetrapyrrole-Based Photodynamic Therapy of Tumors

Norbert Jux and Beate Röder

21. Mechanisms of Cell Death in Photodynamic Therapy David Kessel

22. Photodynamic Therapy and the Tumor Microenvironment Charles J. Gomer, Angela Ferrario, Marian Luna, Natalie Rucker, Sam Wong, Ozguncem Bozkulak and Frank Xu 


\section{Volume 5 Heme Proteins}

23. Hemoproteins Reconstituted with Artificially Created Hemes Takashi Hayashi

24. Tryptophan Catabolism by Heme Dioxygenases Hiroshi Sugimoto, Osamu Takikawa and Yoshitsugu Shiro

25. NO Chemistry by Heme-Enzymes Yoshitsugu Shiro and Shingo Nagano

26. Cytochrome P450 Enzymes Ilia G. Denisov and Stephen G. Sligar

27. Heme Protein-Based Electrochemical Biosensors Genxi Li

28. The Generation and Characterization of the Compounds I and ES States of Cytochrome P450 Using Rapid Mixing Methods

Daniel P. Collins, Tatyana Spolitak, David P. Ballou and John H. Dawson

\section{Volume 6 NMR and EPR Techniques}

29. NMR and EPR Spectroscopy of Paramagnetic Metalloporphyrins and Heme Proteins F. Ann Walker

30. Heme Acquisition by Hemophores: A Lesson from NMR Paola Turano

31. Structure-Function Relationships Among Heme Peroxidases: New Insights from Electronic Absorption, Resonance Raman and Multifrequency Electron Paramagnetic Resonance Spectroscopies Giulietta Smulevich, Alessandro Feis, Barry D. Howes and Anabella Ivancich

\section{Volume 7 Physicochemical Characterization}

32. Electronic and Magnetic Structures of Iron Porphyrin Complexes Mikio Nakamura, Yoshiki Ohgo and Akira Ikezaki

33. Optically Active Porphyrin Systems Analyzed by Circular Dichroism Nagao Kobayashi

34. Photochemical and Photophysical Properties of Metallophthalocyanines Tebello Nyokong and Edith Antunes

35. Structure, Spectroscopy, Photophysics, and Tautomerism of Free-Base Porphycenes and Other Porphyrin Isomers Jacek Waluk 
36. Recent Applications of Infrared Spectroscopy and Microscopy in Chemistry, Biology and Medicine

Petra Hellwig and Frédéric Melin

\section{Volume 8 Open-Chain Oligopyrrole Systems}

37. BODIPY ${ }^{\circledR}$ Dyes and Their Derivatives: Syntheses and Spectroscopic Properties Aurore Loudet and Kevin Burgess

38. Supramolecular Chemistry of Pyrrole-Based $\pi$-Conjugated Acyclic Anion Receptors Hiromitsu Maeda

39. The Synthesis and Properties of Dipyrrins Tabitha E. Wood, Md. Imam Uddin and Alison Thompson

40. Coordination Chemistry of Verdohemes and Open-Chain Oligopyrrole Systems Involved in Heme Oxidation and Porphyrin Destruction Alan L. Balch and Faye L. Bowles

41. Beyond Dipyrrins: Coordination Interactions and Templated Macrocyclizations of Open-Chain Oligopyrroles Martin Bröring

\section{Volume 9 Electronic Absorption Spectra - Phthalocyanines}

42. UV-Visible Absorption Spectroscopic Properties of Phthalocyanines and Related Macrocycles Takamitsu Fukuda and Nagao Kobayashi

\section{Volume 10 Catalysis and Bio-Inspired Systems — Part I}

43. Metalloporphyrin-Catalyzed Asymmetric Atom/Group Transfer Reactions Joshua V. Ruppel, Kimberly B. Fields, Nicole L. Snyder and $X$. Peter Zhang

44. High-Valent Iron-Oxo Porphyrins in Oxygenation Reactions Sam P. de Visser and Wonwoo Nam

45. On the Significance of Phthalocyanines in Solar Cells M. Victoria Martínez-Díaz and Tomás Torres

46. Artificial Photosynthetic Systems Composed of Porphyrins and Phthalocyanines

Shunichi Fukuzumi 
47. Anchoring of Porphyrins and Phthalocyanines on Conductors and Semiconductors for Use in Hybrid Electronics Florence Duclairoir and Jean-Claude Marchon

48. Bioinspired Catalysts with $\mathrm{B}_{12}$ Enzyme Functions Yoshio Hisaeda and Hisashi Shimakoshi

\section{Volume 11 Catalysis and Bio-Inspired Systems — Part II}

49. Bio-inspired Molecular Devices Based on Systems Found in Photosynthetic Bacteria Pierre D. Harvey, Christine Stern and Roger Guilard

50. Self-Assembled Porphyrin Nanostructures Craig J. Medforth and John A. Shelnutt

51. Photochemistry of Chlorophylls and Their Synthetic Analogs Hitoshi Tamiaki and Michio Kunieda

52. Chemistry, Biology and Medical Effects of Water-Soluble Metalloporphyrins Ines Batinić-Haberle, Júlio S. Rebouças, Ludmil Benov and Ivan Spasojević

\section{Volume 12 Applications}

53. Liquid Crystals of Phthalocyanines, Porphyrins and Related Compounds Kazuchika Ohta, Huy-Danh Nguyen-Tran, Lisa Tauchi, Yoriko Kanai, Takashi Megumi and Yasufumi Takagi

54. Porphyrin-Based Chemical Sensors Roberto Paolesse, Donato Monti, Sara Nardis and Corrado Di Natale

55. Electropolymerized Thin Films of Metalloporphyrins for Electrocatalysis and Electroanalysis Sophie Griveau and Fethi Bedioui

56. Application of Porphyrins and Related Compounds as Optical Oxygen Sensors Ichiro Okura and Toshiaki Kamachi

57. Applications of Nanoparticles Containing Porphyrins and Related Systems Sara Bonacchi, Damiano Genovese, Riccardo Juris, Fabrizio Mancin, Marco Montalti, Luca Prodi, Enrico Rampazzo and Nelsi Zaccheroni 


\section{Volume 13 Synthesis and Structural Studies}

58. The Synthesis of $\pi$-Extended Porphyrins Andrei V. Cheprakov

59. Noncovalent Syntheses of Multiporphyrin Species in Aqueous Solution Rosaria Lauceri, Alessandro D'urso and Roberto Purrello

60. Porphyrins and Porphyrin Analogs Bearing Double Bonds at One or More Meso Positions

Chang-Hee Lee, Vladimir Roznyatovskiy, Seong-Jin Hong and Jonathan L. Sessler

61. The Structural Chemistry of Isolated Chlorophylls Mathias O. Senge and Stuart A. MacGowan

62. Porphyrin-Based Tectons in Molecular Tectonics Véronique Bulach and Mir Wais Hosseini

\section{Volume 14 Theoretical, Electron Transfer and Physical Studies}

63. The Role of Heme-Nitrosyls in the Biosynthesis, Transport, Sensing, and Detoxification of Nitric Oxide (NO) in Biological Systems: Enzymes and Model Complexes

Nicolai Lehnert, Timothy C. Berto, Mary Grace I. Galinato and Lauren E. Goodrich

64. Chemistry of Sandwich Tetrapyrrole Rare Earth Complexes Yongzhong Bian, Yuexing Zhang, Zhongping Ou and Jianzhuang Jiang

65. Theoretical Aspects of the Optical Spectroscopy of Porphyrinoids Martin J. Stillman

66. Synthesis and Reactivity of High-Valent Transition Metal Corroles and Corrolazines Amanda J. McGown, Yosra M. Badiei, Pannee Leeladee, Katharine A. Prokop, Serena DeBeer and David P. Goldberg

\section{Volume 15 Biochemistry of Tetrapyrroles}

67. Eukaryotic Heme Trafficking Scott Severance, Caiyong Chen and Iqbal Hamza

68. Ferrochelatase Structure and Reaction Mechanism Gloria C. Ferreira and Gregory A. Hunter

69. Heme Proteins as Gas Sensors Emily E. Weinert and Michael A. Marletta 
70. Heme Biosynthesis

Gunhild Layer, Dieter Jahn and Martina Jahn

71. Mechanisms of Cytoprotection by Hemopexin Ann Smith

72. Mechanisms of Heme Uptake and Utilization in Bacterial Pathogens Angela Wilks and Kylie D. Barker

73. Emerging Roles of Heme as a Signal and a Gas-Sensing Site: Heme-Sensing and Gas-Sensing Proteins Jotaro Igarashi, Kenichi Kitanishi and Toru Shimizu

\section{Volume 16 Synthetic Developments - Part I}

74. Carbaporphyrins and Related Systems. Synthesis, Characterization, Reactivity and Insights into Porphyrinoid Aromaticity Timothy D. Lash

75. Synthesis and Properties of the Hybrid PhthalocyanineTetrabenzoporphyrin Macrocycles Andrew N. Cammidge, Isabelle Chambrier, Michael J. Cook and Lydia Sosa-Vargas

\section{Volume 17 Synthetic Developments - Part II}

76. Syntheses of Bacteriochlorins and Isobacteriochlorins Christian Brückner, Lalith Samankumara and Junichi Ogikubo

77. The Hemiporphyrazines and Related Systems Christopher J. Ziegler

78. Electrochemistry of Pyrroles and Oligopyrroles: Analytic and Synthetic Aspects Christophe Bucher and Mihai Buda

\section{Volume 18 Applications and Materials}

79. Ordered Surface Structures of Self-Assembled Porphyrins Nico Veling, Johannes A. A. W. Elemans, Roeland J. M. Nolte and Alan E. Rowan

80. Porphyrin- and Phthalocyanine-Based Solar Cells Hiroshi Imahori, Kei Kurotobi, Michael G. Walter, Alexander B. Rudine and Carl C. Wamser

81. Structures and Properties of Non-Planar Tetrapyrroles Jonathan P. Hill, Shinsuke Ishihara and Katsuhiko Ariga 
82. Porphyrin-Based Nanocomposites for Biosensing Jianping Lei and Huangxian Ju

83. Electrochemical Organic Electronics Based On Robust Thin-Films of Porphyrazines

Simon Dalgleish and Kunio Awaga

84. Bioconjugation of Phthalocyanine Derivatives Pui-Chi Lo, Janet T. F. Lau and Dennis K. P. Ng

85. Transition Metal Complexes of Porphyrins and Porphyrinoids Andrey Mironov

\section{Volume 19 Biochemistry of Tetrapyrroles - Part II}

86. Biochemistry of Methyl-CoM Reductase and Coenzyme $\mathrm{F}_{430}$ Yuzhen Zhou, Dariusz Sliwa and Stephen Ragsdale

87. Peroxidase and Heme Thiolate Enzymes Thomas L. Poulos

88. Biosynthesis of Siroheme, Coenzyme $\mathrm{F}_{430}$ and Heme $d_{1}$ Gunhild Layer and Martin J. Warren

89. Diversity of Heme Proteins in Sulfate Reducing Bacteria Célia V. Romão, Margarida Archer, Susana A. Lobo, Ricardo O. Louro, Inês A. C. Pereira, Lígia M. Saraiva, Miguel Teixeira and Pedro M. Matias

90. Dioxygen-Generating Chlorite Dismutases and the CDE Protein Superfamily Jennifer L. DuBois and Jeffery A. Mayfield

91. Side Chain Modification During Heme Biosynthesis John D. Phillips

92. The Cytochrome $b_{561}$ Protein Family Shin-ichi J. Takayama and A.Grant Mauk

93. Heme Attachment to Cytochromes $c$ Julie M. Stevens and Stuart J. Ferguson

\section{Volume 20 Chlorophylls and Related Systems}

94. Key Enzymes of Chlorophyll Biosynthesis Markus J. Broecker, Dieter Jahn and Jürgen Moser

95. Supramolecular Chlorophyll Assemblies for Artificial Photosynthesis Victoria L. Gunderson and Michael R. Wasielewski

96. Bacteriochlorophyll Biosynthesis and Assembly in Green Chlorophototrophic Bacteria: Theme and Variations Zhenfeng Liu and Donald Bryant 
97. Tetrapyrrole Biosynthesis in Plant Systems Tatsuru Masuda and Koichi Kobayashi

98. Chlorophyll Metabolism in Photosynthetic Organisms Ryouich Tanaka, Atsushi Takabayashi, Hisashi Ito and Ayumi Tanaka

\section{Volume 21 Catalysis}

99. Porphyrin and Corrole Platforms for Water Oxidation, Oxygen Reduction, and Peroxide Dismutation

Christopher M. Lemon, Dilek K. Dogutan and Daniel G. Nocera

100. An Overview of Metalloporphyrin-Catalyzed Carbon and Nitrogen Group Transfer Reactions Bernie J. Anding and L. Keith Woo

101. Metalloporphyrin Catalyzed C-C Bond Formation Cong-Ying Zhou, Vanessa Kar-Yan Lo and Chi-Ming Che

102. Recent Advances in Catalysis by Water-Soluble Metalloporphyrins Gérard Simonneaux, Paul Le Maux, Soizic Chevance and Hassan Srour

\section{Volume 22 Biophysical and Physicochemical Studies of Tetrapyrroles}

103. Phycobiliproteins Kai-Hong Zhao, Robert J. Porra and Hugo Scheer

104. Formation Principles and Excited States Relaxation in Self-Assembled Complexes: Multiporphyrin Arrays and "Semiconductor CdSe/ZnS Quantum Dot-Porphyrin" Nanocomposites Eduard I. Zenkevich and Christian W. von Borczyskowski

105. Quantum Chemical Studies on the Excited-State Deactivation Mechanism in Transition-Metal Tetrapyrroles Angela Rosa and Giampaolo Ricciardi

106. Hemoprotein Models

Richard A. Decréau

\section{Volume 23 Synthesis}

107. Enumeration of Isomers of Substituted Tetrapyrrole Macrocycles: From Classical Problems in Biology to Modern Combinatorial Libraries Masahiko Taniguchi and Jonathan S. Lindsey

108. Survey of Synthetic Routes for Synthesis and Substitution in Porphyrins Irina P. Beletskaya, Vladimir S. Tyurin, Alexei Uglov, Christine Stern and Roger Guilard 
109. The Effect of Structural Modifications on the Properties of Porphyrinoids John Mack, Nagao Kobayashi and Zhen Shen

110. Synthesis and Characterization of Chiral Phthalocyanines Nagao Kobayashi

\section{Volume 24 Coordination Chemistry and Materials}

111. Stereochemical Systematics for Porphyrins and Metalloporphyrins W. Robert Scheidt

112. Coordination Chemistry of Crown Ether-Porphyrin Conjugates Norbert Jux

113. Crown-Substituted Phthalocyanines: From Synthesis Towards Materials Yulia G. Gorbunova, Alexander G. Martynov and Aslan Yu. Tsivadze

114. Phthalocyanines as Sensitizers in Dye-Sensitized Solar Cells Derck Schlettwein and Tebello Nyokong

\section{Volume 25 Vitamin B12 and Related Systems}

115. Biosynthesis of Vitamin B12 Finian J. Leeper, Martin J. Warren, James M. Kelly and Andrew D. Lawrence

116. Vitamin B12 Derivatives for Spectroanalytical and Medicinal Applications Felix Zelder and Roger Alberto

117. Vitamin B12-Derivatives: Organometallic Catalysts, Cofactors and Ligands of Bio-Macromolecules Bernhard Kräutler and Barbara Puffer

118. Chemical Synthesis of Artificial Corrins Franz-Peter Montforts, Martina Osmers and Dennis Leupold

\section{Volume 26 Heme Biochemistry}

119. Toward Heme: 5-Aminolevulinate Synthase and Initiation of Porphyrin Synthesis

Erica J. Fratz, Bosko M. Stojanovski and Gloria C. Ferreira

120. The Dance of Porphobilinogen Synthase in the Control of Tetrapyrrole Biosynthesis

Eileen K. Jaffe and Sarah H. Lawrence

121. The Ultimate Step of Heme Biosynthesis: Orchestration Between Iron Trafficking and Porphyrin Synthesis Mallory E. Gillam, Gregory A. Hunter and Gloria C. Ferreira 
122. Heme-Delivering Proteins in Bacteria Cécile Wandersman and Philippe Delepelaire

123. Porphyrin and Heme Trafficking in Metazoans Iqbal Hamza and Harry A. Dailey

124. Extracellular Heme Uptake and Metabolism in Bacterial Pathogenesis Angela Wilks and Maura J. O'Neill

125. Heme and Infectious Diseases Allison J. Farrand and Eric P. Skaar

126. Biliverdin Reductase: Its Multiple Functions in Cell Signaling and Role in Cytoprotection

Mahin D. Maines and Peter E.M. Gibbs

127. Reactivity and Structure in the CDE Protein Superfamily: From $\mathrm{O}_{2}$ Generation to Peroxidase Chemistry and Beyond Jennifer L. DuBois, Christopher J. Carrell and Carrie M. Wilmot

\section{Volume 27 Erythropoiesis, Heme and Applications to Biomedicine}

128. Differential Regulation of 5-Aminolevulinate Synthase Isozymes in Vertebrates Kazumichi Furuyama and Masayuki Yamamoto

129. Erythropoiesis, Hemoglobin Synthesis, and Erythroid Mitochondrial Iron Homeostasis Prem Ponka, Mark J. Koury and Alex D. Sheftel

130. Zebrafish as a Genetic Model of Hematopoiesis and Human Red Blood Cell Diseases Dhvanit I. Shah and Barry H. Paw

131. Hemoglobin and Hemoglobin-based Oxygen Carriers: A Biochemical, Pharmacological, and Toxicological Perspective Yiping Jia, Claudia P. Pereira and Paul W. Buehler

132. Unique Features of Heme Biosynthesis in the Malaria Parasite Govindarajan Padmanaban, V. Arun Nagaraj and Pundi N. Rangarajan

133. Iron(III) Protoporphyrin IX and Hemozoin: Key Targets in the Chemotherapy of Malaria Katherine A. de Villiers and Timothy J. Egan

134. Cyclic Tetrapyrroles in Photodynamic Therapy: The Chemistry of Porphyrins and Related Compounds in Medicine Tyler G. St. Denis, Ying-Ying Huang and Michael R. Hamblin

135. Porphyrin Conjugates for Cancer Therapy Francesca Giuntini, Ross Boyle, Martha Sibrian-Vazquez and M. Graça H. Vicente 


\section{Volume 28 Chlorophyll, Photosynthesis and Bio-inspired Energy}

136. Structural Analysis of Photosynthetic Proteins

Christopher Kupitz, Raimund Fromme, Ingo Grotjohann and Petra Fromme

137. Magnesium Chelatase: The Molecular Motor of Chlorophyll Biosynthesis

Mats Hansson, Joakim Lundqvist, Nick Sirijovski and Salam Al-Karadaghi

138. The Magnesium Branch of Tetrapyrrole Biosynthesis: From Magnesium Protoporphyrin IX to Chlorophyll a/b Annabel Salinas and Bernhard Grimm

139. Chlorophyll Breakdown: Chemistry, Biochemistry, and Biology Bernhard Kräutler and Stefan Hörtensteiner

140. Phycobiliproteins - Biosynthesis, Assembly and Applications Kristina E. Overkamp and Nicole Frankenberg-Dinkel

141. Binary Ionic Porphyrin Nanomaterials for Energy from Sunlight John A. Shelnutt, Yongming Tian, Kathleen E. Martin and Craig J. Medforth

142. Porphyrin-Sensitized Solar Cells Eric Wei-Guang Diau and Lu-Lin Li

\section{Volume 29 Porphyrias and Sideroblastic Anemias}

143. The Structural Basis of Porphyrias - Defects of Heme Biosynthetic Enzymes Anna-Lena Kaufholz, Gunhild Layer, Dirk Heinz, Martina Jahn and Dieter Jahn

144. Sideroblastic Anemias: Molecular Basis, Pathophysiology, and Clinical Aspects Sylvia S. Bottomley and Mark D. Fleming

145. Heme Biosynthesis and Pathophysiology of Porphyrias Zoubida Karim, Laurent Gouya, Jean-Charles Deybach and Hervé Puy

146. Clinical and Molecular Epidemiology of the Porphyrias Michael N. Badminton, George H. Elder and Sharon D. Whatley

147. Congenital Erythropoietic Porphyria Jordi To-Figueras, Oscar Millet and Carmen Herrero

148. Porphyria Cutanea Tarda Ashwani K. Singal and John D. Phillips 
149. Variegate Porphyria

Peter Meissner, Anne Corrigall and Richard Hift

150. Erythropoietic Protoporphyria and X-Linked Dominant Protoporphyria Xiaoye Schneider-Yin and Elisabeth I. Minder

151. Liver Transplantation in Acute Hepatic Porphyria and Erythropoietic Protoporphyria

Staffan Wahlin and Pauline Harper

152. Clinical and Laboratory Diagnosis of the Porphyrias Karl E. Anderson

153. Liver Gene Therapy Approaches for Acute Intermittent Porphyria: Metabolic Correction and Immunological Hurdles Antonio Fontanellas and Ignacio Melero

154. Cellular and Gene Therapy for Erythropoietic Porphyrias Emmanuel Richard, Cécile Ged, Aurélie Bedel, François Moreau-Gaudry and Hubert de Verneuil

\section{Volume 30 Heme Proteins - Part II}

155. Porphyrin Modifications in the Autocatalytic Maturation of Hemoproteins Paul R. Ortiz de Montellano

156. Thiol/Disulfide Redox Switches as a Regulatory Mechanism in Heme-binding Proteins Stephen W. Ragsdale, Nirupama Gupta, Ireena Bagai, Andrea Morris Spencer and Eric Carter

157. Non-Canonical Heme-Binding Proteins Luciana Hannibal and Dennis J. Stuehr

158. Heme-Regulated Inhibitor (HRI) eIF2 $\alpha$ Kinase and the Heme-Regulated Turnover of Cytochromes P450 and Other Hepatic Proteins Maria Almira Correia, Yi Liu, YongQiang Wang, Mingxiang Liao, Juan Engel and Shenheng Guan

159. Bacterioferritin: Structure Function and Protein-Protein Interactions Mario Rivera

160. Nuclear Magnetic Resonance as a Tool to Characterize the Interactome of Heme Proteins Daniela Lalli and Paola Turano

161. Mimicking Heme Enzymes in the Solid State Randy W. Larsen 
162. Biophysical Perspectives on the Acquisition, Transport, and Trafficking of Heme in Bacteria

Kenton R. Rodgers and Gudrun S. Lukat-Rodgers

163. Protection against Heme Toxicity: Hemopexin Rules, OK?

Ann Smith 